\title{
ON THE CLASSIFICATION OF WEAKLY INTEGRAL MODULAR CATEGORIES
}

\author{
PAUL BRUILLARD, CÉSAR GALINDO, SIU-HUNG NG, JULIA Y. PLAVNIK, \\ ERIC C. ROWELL, AND ZHENGHAN WANG
}

\begin{abstract}
We classify all modular categories of dimension $4 m$, where $m$ is an odd square-free integer, and all ranks 6 and 7 weakly integral modular categories. This completes the classification of weakly integral modular categories through rank 7 . Our results imply that all integral modular categories of rank at most 7 are pointed (that is, every simple object has dimension 1). All strictly weakly integral (weakly integral but non-integral) modular categories of ranks 6 and 7 have dimension $4 m$, with $m$ an odd square free integer, so their classification is an application of our main result. The classification of rank 7 integral modular categories is facilitated by an analysis of actions on modular categories by two groups: the Galois group of the field generated by the entries of the $S$-matrix and the group of isomorphism classes of invertible simple objects. The interplay of these two actions is of independent interest, and we derive some valuable arithmetic consequences from their actions.
\end{abstract}

\section{INTRODUCTION}

A modular category over $\mathbb{C}$ is a non-degenerate braided spherical fusion category over $\mathbb{C}$. In this article, we are interested in extending the classification of modular categories over $\mathbb{C}$ of low rank, under some additional constraints on the dimensions of simple objects. A fusion category $\mathcal{C}$ is called integral if the Frobenius-Perron dimensions $\mathrm{FPdim}(X)$ of simple objects $X$ are integers, whereas $\mathcal{C}$ is weakly integral if $\operatorname{FPdim}(X)^{2} \in \mathbb{Z}$ for all simple objects $X$. We completely classify weakly integral categories when the number of simple objects (up to isomorphism) is 6 or 7 . This is facilitated by a more general

Date: October 20, 2015.

The results obtained in this paper were mostly obtained while all 6 authors were at the American Institute of Mathematics, participating in a SQuaRE. We would like to thank that institution for their hospitality and encouragement. C. Galindo was partially supported by the FAPA funds from vicerrectoria de investigaciones de la Universidad de los Andes, S.-H. Ng by NSF DMS-1303253 and DMS-1501179, J.Plavnik by CONICET, ANPCyT and Secyt-UNC, E. Rowell by NSF grant DMS1108725, and Z. Wang by NSF grant DMS-1108736. PNNL-SA-110195. This project began while J.P. was at Universidad de Buenos Aires, and the support of that institution is gratefully acknowledged. 
theorem in which we classify modular categories of dimension $4 m$, where $m$ is an odd square-free integer.

Broad classes of integral modular categories arise from finite groups, for instance the representation categories $\operatorname{Rep}\left(D^{\omega} G\right)$ of twisted Drinfeld doubles $D^{\omega} G$ of finite groups. Known constructions of weakly integral modular categories that are not integral (called strictly weakly integral) are somewhat fewer. Common examples are the modular categories $S O(N)_{2}$ associated with quantum groups $U_{q} \mathfrak{s o}_{N}$ at $q=e^{\pi i /(2 N)}$, for $N$ odd and $q=e^{\pi i / N}$, for $N$ even (see e.g. [26]), Drinfeld centers of weakly integral modular categories and $\mathbb{Z}_{2}$-equivariantizations (see [18] and [14]) of the braided $\mathbb{Z}_{2}$-crossed fusion category structure of Tambara-Yamagami categories [32] associated to a group $A$ of odd order. Indeed, by the proof of Theorem 3.1, any strictly weakly integral modular category of dimension $4 k$ with $k>1$ an odd square-free integer and with exactly two isomorphism classes of invertible objects must be of the form $T Y\left(\mathbb{Z}_{k}, \chi, \nu\right)^{\mathbb{Z}_{2}}$, as a braided fusion category.

Besides the application to low rank classification, a referee pointed out the following compelling reason to study modular categories with (nearly) square-free dimensions: these lie in non-trivial Witt classes [10]. The trivial Witt class consists of non-degenerate categories obtained as Drinfeld centers of fusion categories. Any weakly integral category in this class must have dimension $n^{2}$ for some $n \in \mathbb{Z}$. Studying modular categories with dimension $n^{2}$ is therefore at least as hard as studying weakly integral fusion categories of dimension $n$.

The $\mathbb{Z}_{2}$-equivariantization mentioned above is a special case of a general method, called gauging [2,8], for producing new weakly integral modular categories. Given a weakly integral modular category $\mathcal{C}$ with a categorical action (or symmetry) of a finite group $G$, then gauging the $G$ symmetry of $\mathcal{C}$ as defined in $[2,8]$ results in a weakly integral modular category denoted $\mathcal{C} / / G$. An interesting weakly integral modular category is obtained by gauging the $S_{3}$ symmetry of $S O(8)_{1}$ in [8] described as Example J in [2]. While representations of the braid groups from weakly integral modular categories are all conjectured to have finite images [26], they are still useful for topological quantum computation [26]. Actually, weakly integral modular categories are potentially more easily realized in nature than non-weakly integral categories. Moreover, they can be made universal for quantum computation in some cases such as the metaplectic modular categories [9].

The following classes of weakly integral modular categories appear in our classification:

(i) A pointed modular category is a modular category in which all simple objects are invertible, that is, $X \otimes X^{*} \cong 1$. Such categories have the same fusion 
rules as $\operatorname{Rep}(A)$ where $A$ is a finite abelian group. A cyclic modular category is a pointed modular category with the same fusion rules as $\operatorname{Rep}\left(\mathbb{Z}_{n}\right)$.

(ii) An Ising modular category $\mathcal{I}$ is a non-pointed modular category with $\operatorname{dim} \mathcal{I}=4$ (see [13, Appendix B] where such categories are classified). Such a category has rank 3 with two simple classes of dimension 1 and one of dimension $\sqrt{2}$.

(iii) A metaplectic modular category $\mathcal{C}$ is any modular category with the same fusion rules as $S O(N)_{2}$ for $N$ odd (see e.g. see [25]). These rank $\frac{N+7}{2}, 4 N-$ dimensional categories have two 1-dimensional objects and two objects of dimension $\sqrt{N}$, while the remaining $\frac{N-1}{2}$ objects have dimension 2 . For example, $T Y\left(\mathbb{Z}_{N}, \chi, \nu\right)^{\mathbb{Z}_{2}}$ for $N$ odd is a metaplectic modular category (see [18]).

Remark 1.1. There are exactly eight unitary Ising categories [21]. The Ising theory refers to the one with central charge $c=\frac{1}{2}$ and the topological twist of the non-pointed simple object is $\theta=e^{\frac{2 \pi i}{16}}$.

Our results are summarized in:

Theorem 1.2. Suppose that $\mathcal{C}$ is a weakly integral modular category either of rank $\leq 7$ or with $\mathrm{FPdim}(\mathcal{C})=4 m$, where $m$ is an odd square-free integer. Then $\mathcal{C}$ is equivalent to a (Deligne) product of the following: pointed categories, Ising categories and metaplectic categories.

Our approach involves a number of well-established classification techniques as well as some new ones. A central theme will be the application of group actions on modular categories $\mathcal{C}$. In particular, two groups act on equivalence classes $\operatorname{Irr}(\mathcal{C})$ of simple objects: the Galois group $\operatorname{Gal}(\mathcal{C})$ associated to the splitting field of the Grothendieck ring $K_{0}(\mathcal{C})$ and the group $G(\mathcal{C})$ of invertible objects which is nontrivial for strictly weakly integral modular categories. We study the relationship between these two group actions as well as their structures, which yield some useful constraints on the dimension of the classified categories.

\section{Preliminaries}

In this section, we set the notation and recall some useful results from the literature. Of particular importance are the groups $G(\mathcal{C})$ of isomorphism classes of invertible objects and $\operatorname{Gal}(\mathcal{C})$ the Galois group of the number field generated by the entries of the $S$ matrix.

2.1 Fusion categories By a fusion category we mean $\mathbb{C}$-linear semisimple rigid tensor category with finitely many isomorphism classes of simple objects and simple unit object 1 . We denote by $\operatorname{Irr}(\mathcal{C})$ the set of isomorphism class of simple objects of $\mathcal{C}$. A 
representative of $i \in \operatorname{Irr}(\mathcal{C})$ will be denoted by $X_{i}$ and the isomorphism class of the unit object 1 will be labelled $0 \in \operatorname{Irr}(\mathcal{C})$. For further reading we direct the reader to [1,17].

The set of isomorphism classes of invertible objects of a fusion category $\mathcal{C}$ forms a group, denoted $G(\mathcal{C})$, with multiplication induced by the tensor product. A fusion category is called pointed if all its simple objects are multiplicatively invertible. Every pointed fusion category is tensor equivalent to $\operatorname{Vec}_{G}^{\omega}$, that is, the category of $G$-graded finite dimensional vector spaces, where $G=G(\mathcal{C})$ and the associativity constraint is given by a 3-cocycle $\omega \in Z^{3}\left(G, \mathbb{C}^{*}\right)$. By a fusion subcategory of a fusion category we mean a full abelian $\mathbb{C}$-linear tensor subcategory. For a fusion category $\mathcal{C}$, we denote by $\mathcal{C}_{p t}$ the fusion subcategory generated by the invertible objects of $\mathcal{C}$.

The Grothendieck ring $K_{0}(\mathcal{C})$ of a fusion category $\mathcal{C}$ has $\mathbb{Z}$-basis $\operatorname{Irr}(\mathcal{C})$ with multiplication $X_{i} X_{j}=\sum_{k \in \operatorname{Irr}(\mathcal{C})} N_{i j}^{k} X_{k}$, where $N_{i j}^{k}:=\operatorname{dim} \operatorname{Hom}\left(X_{i} \otimes X_{j}, X_{k}\right)$. Defining $\left(N_{i}\right)_{k, j}=N_{i, j}^{k}$ we obtain a representation of the Grothendieck ring via $X_{i} \rightarrow N_{i}$ by extending linearly. The Frobenius-Perron dimension FPdim $(X)$ is defined as the largest eigenvalue of $N_{X}$, and $\operatorname{FPdim}(\mathcal{C})=\sum_{i \in \operatorname{Irr}(\mathcal{C})} \operatorname{FPdim}\left(X_{i}\right)^{2}$ (see 17$]$ ). We will say $\mathcal{C}$ is weakly integral if $\mathrm{FPdim}(\mathcal{C}) \in \mathbb{Z}$ and strictly weakly integral if it is weakly integral and has a simple object of non-integral dimension.

2.2 Spherical structures and categorical dimension A spherical structure on a fusion category $\mathcal{C}$ is a tensor isomorphism $\psi: \operatorname{Id}_{\mathcal{C}} \rightarrow(-)^{* *}$ such that $d_{+}(X)=d_{-}(X)$ for all $X \in \operatorname{Irr} \mathcal{C}$, where

$$
\begin{aligned}
& d_{+}(X): \mathbf{1} \stackrel{\operatorname{coev}_{X}}{\longrightarrow} X \otimes X^{*} \stackrel{\psi_{X} \otimes \mathrm{id}_{X^{*}}}{\longrightarrow} X^{* *} \otimes X^{*} \stackrel{\mathrm{ev}_{X^{*}}}{\longrightarrow} 1, \\
& d_{-}(X): \mathbf{1} \stackrel{\operatorname{coev}_{X^{*}}}{\longrightarrow} X^{*} \otimes X^{* *} \stackrel{\operatorname{id}_{X^{*}} \otimes \psi_{X}^{-1}}{\longrightarrow} X^{*} \otimes X \stackrel{\mathrm{ev}_{X}}{\longrightarrow} 1 .
\end{aligned}
$$

In a spherical category we can define a tracial map $\operatorname{Tr}: \operatorname{End}_{\mathcal{C}}(X) \rightarrow \mathbb{C}$ and we have $d(X):=\operatorname{Tr}\left(\operatorname{id}_{X}\right)=d_{+}(X)=d_{-}(X)$. The number $d(X)$ is called the categorical dimension of $X$, and $\operatorname{dim}(\mathcal{C})=\sum_{i \in \operatorname{Irr}(\mathcal{C})} d\left(X_{i}\right)^{2}$ is called the global dimension of $\mathcal{C}$. Moreover, the map $d: K_{0}(\mathcal{C}) \rightarrow \mathbb{C}$ is a ring homomorphism. A fusion category is called pseudo-unitary if there is a spherical structure such that $\operatorname{dim}(\mathcal{C})=\operatorname{FPdim}(\mathcal{C}),[17$.

2.3 Modular Categories and Modular Data A braided fusion category $(\mathcal{C}, c)$ is a fusion category $\mathcal{C}$ with a braiding $c_{X, Y}: X \otimes Y \rightarrow Y \otimes X$, see [1]. A ribbon structure on a braided fusion category $\mathcal{C}$ is a natural automorphism $\theta \in \operatorname{Aut}\left(\operatorname{Id}_{\mathcal{C}}\right)$ of the identity functor such that

$$
\theta_{X \otimes Y}=\theta_{X} \otimes \theta_{Y} c_{X, Y} c_{Y, X}, \quad \theta_{X}^{*}=\theta_{X^{*}} .
$$

There is a one-to-one correspondence between ribbon structures over a braided fusion categories $\mathcal{C}$ and spherical structures over $\mathcal{C},[14]$. 
A premodular category $\mathcal{C}$ (or alternatively a ribbon fusion category) is a braided fusion category with a spherical structure. A premodular cateogry $\mathcal{C}$ is called modular if the (unnormalized) $S$-matrix, defined as $S_{i j}=\operatorname{Tr}\left(c_{X_{j}, X_{i}^{*}} c_{X_{i}^{*}, X_{j}}\right)$ is non-singular. The $S$-matrix is symmetric and $S_{0, i}=d\left(X_{i}\right)$.

Let $\mathcal{C}$ be a modular category with associated ribbon structure $\theta$. The twists are $\theta_{i}:=\theta_{X_{i}}$. The (unnormalized) $T$-matrix of $\mathcal{C}$ is defined by $T_{i j}=\delta_{i j} \theta_{i}$ and has finite order (cf. [1]). The order of $T$ is equal to the Frobenius-Schur exponent of $\mathcal{C}$ (see [27] for more details) denoted $\mathrm{FS} \exp (\mathcal{C})$. The Gauss sums $p_{ \pm}:=\sum_{i \in \operatorname{Irr}(\mathcal{C})} d_{i}^{2} \theta_{i}^{ \pm 1}$ satisfy

$p_{+} p_{-}=\operatorname{dim} \mathcal{C}$ and $\left(\frac{p_{+}}{p_{-}}\right)^{2 \mathrm{FS} \exp (\mathcal{C})}=1$. The pair $(S, T)$ is called the modular data of $\mathcal{C}$, and it satisfies the following equations (see [5, Sect. 2] or [1, 3.1]).

(i) Twist equation:

$$
p_{+} S_{i j}=\theta_{i} \theta_{j} \sum_{k} S_{k i} S_{k j} \theta_{k}
$$

(ii) Balancing equation:

$$
S_{i j} \theta_{i} \theta_{j}=\sum_{k} N_{i^{*} j}^{k} d_{k} \theta_{k}
$$

(iii) Orthogonality:

$$
S S^{\dagger}=\operatorname{dim}(\mathcal{C}) I
$$

where $\dagger$ is the conjugate-transpose operation and $I$ denotes the identity matrix.

The twist equation comes from the relation $(S T)^{3}=p_{+} S^{2}$. While $(S, T)$ give rise to a projective representation of the modular group $\mathrm{SL}(2, \mathbb{Z})$, one may normalize $s=S / x$, $t=T / y$ so that $(s, t)$ gives a linear representation of $\mathrm{SL}(2, \mathbb{Z})$. We call a pair $(s, t)$ obtained in this way a normalized modular data for $\mathcal{C}$.

2.4 The Müger Center Two objects $X$ and $Y$ of a braided fusion category $(\mathcal{C}, c)$ are said to centralize each other if $c_{Y, X} c_{X, Y}=\mathrm{id}{ }_{X \otimes Y}$. Müger has shown that $X$ and $Y$ centralize each other if and only if $S_{X Y}=d_{X} d_{Y}$ [23, Prop. 2.5]. The centralizer $\mathcal{D}^{\prime}$ of a fusion subcategory $\mathcal{D} \subseteq \mathcal{C}$ is defined to be the full subcategory of objects of $\mathcal{C}$ that centralize every object of $\mathcal{D}$, that is

$$
\mathcal{D}^{\prime}=\left\{X \in \mathcal{C} \mid c_{Y, X} c_{X, Y}=\operatorname{id}_{X \otimes Y} \text {, for all } Y \in \mathcal{D}\right\} .
$$

The Müger center $Z_{2}(\mathcal{C})$ of a braided fusion category $\mathcal{C}$ is the centralizer of $\mathcal{C}$, that is $Z_{2}(\mathcal{C})=\mathcal{C}^{\prime}$. A braided fusion category $\mathcal{C}$ is called symmetric when $Z_{2}(\mathcal{C})=\mathcal{C}$, so in particular the Müger center of a braided fusion category is a symmetric fusion 
subcategory. A symmetric fusion category $\mathcal{C}$ is called Tannakian if admits a fiber functor, that is, a symmetric functor $\mathcal{C} \rightarrow$ Vec.

A braided fusion category is non-degenerate if $Z_{2}(\mathcal{C}) \cong$ Vec. For premodular categories, non-degeneracy is equivalent to modularity, i.e. the invertibility of the $S$-matrix $([3])$.

If $\mathcal{D} \subset \mathcal{C}$ are both modular, then the centralizer $\mathcal{D}^{\prime}$ is also modular [23]. In particular, one has $\mathcal{C} \cong \mathcal{D} \otimes \mathcal{D}^{\prime}$ where $\otimes$ denotes the Deligne product, see $[11]$. In general $\mathcal{A} \otimes \mathcal{B}$ has rank $a b$ where the ranks of $\mathcal{A}$ and $\mathcal{B}$ are $a$ and $b$, respectively.

2.5 $G$-grading A fusion category $\mathcal{C}$ is $G$-graded ( $G$ a finite group) if $\mathcal{C}=\bigoplus_{g \in \Gamma} \mathcal{C}_{g}$ as an abelian category and $\mathcal{C}_{g} \otimes \mathcal{C}_{h} \subset \mathcal{C}_{g h}$. If each $\mathcal{C}_{g}$ is non-empty, the grading is called faithful. It was proved in [19, Theorem 3.5] that any fusion category $\mathcal{C}$ is naturally graded by a group $U(\mathcal{C})$, called the universal grading group of $\mathcal{C}$, and the adjoint subcategory $\mathcal{C}_{a d}$ (generated by all subobjects of $X^{*} \otimes X$, for all $X$ ) is the trivial component of this grading. Moreover, any other faithful grading of $\mathcal{C}$ arises from a quotient of $U(\mathcal{C})$ [19, Corollary 3.7].

A strictly weakly integral fusion category is faithfully graded by an elementary abelian 2-group 19, Theorem 3.10]. Indeed, as each simple object has dimension $\sqrt{k}$, for some $k \in \mathbb{Z}$, one may partition the simple objects into finitely many non-empty sets of the form $A_{n}:=\left\{X: d_{X} \in \sqrt{n} \mathbb{Z}\right\}$, where $n$ is square-free, and this partition induces a faithful grading by an elementary 2-group. The trivial component $\mathcal{C}_{e}$ with respect to this grading is the subcategory $\mathcal{C}_{\text {int }}$ generated by the simple objects of $\mathcal{C}$ of integral dimension.

For any abelian group $A$, let denote $\hat{A}$ the abelian group of linear complex characters. For a braided fusion category, there is group homomorphism $\chi: U(\mathcal{C}) \rightarrow \widehat{G(\mathcal{C})}$, roughly defined as follows: For $g \in G(\mathcal{C})$ and $i \in \operatorname{Irr} \mathcal{C}$ the double braiding $c_{i, g} c_{g, i}$ is an isomorphism on the simple object $g \otimes i$, and hence a scalar map $\chi(i, g) I d_{g \otimes i}$. It can be shown that for each $i, \chi(i,-)$ is a character. Therefore we obtain a multiplicative map $\chi: K_{0}(\mathcal{C}) \rightarrow \widehat{G(\mathcal{C})}$ and this map induces a group homomorphism $\chi: U(\mathcal{C}) \rightarrow \widehat{G(\mathcal{C})}$. Now, if $\mathcal{C}$ is modular $\chi$ is an isomorphism 19, Theorem 6.2]. Observe that $A \cong \hat{A}$ (non-canonically) for any abelian group, $U(\mathcal{C}) \cong G(\mathcal{C})$ for modular categories.

2.6 Galois Group Action Let $\mathcal{C}$ be a modular category with modular data $(S, T)$. We define the Galois group $\operatorname{Gal}(\mathcal{C})$ of $\mathcal{C}$ as $\operatorname{Gal}(\mathcal{C}):=\operatorname{Gal}\left(\mathbb{K}_{\mathcal{C}} / \mathbb{Q}\right)$, where $\mathbb{K}_{\mathcal{C}}=\mathbb{Q}\left(S_{i j} \mid\right.$ $i, j \in \operatorname{Irr}(\mathcal{C}))$. Notice that $\mathbb{K}_{\mathcal{C}}$ is the splitting field of the set of characteristic polynomials of the fusion matrices $N_{i}$. The action of $\operatorname{Gal}(\mathcal{C})$ on the normalized columns with $i$ th 
entry $S_{i j} / d_{j}$ of the $S$-matrix induces a faithful action on $\operatorname{Irr}(\mathcal{C})$ (cf. [5]). We will denote by $\hat{\sigma}$ the permutation on $\operatorname{Irr}(\mathcal{C})$ associated with $\sigma \in \operatorname{Gal}(\mathcal{C})$.

Let $\zeta_{N}=e^{\frac{2 \pi i}{N}}$ where $N=\operatorname{FSexp}(\mathcal{C})$, then we have the exact sequence:

$$
1 \rightarrow \operatorname{Gal}\left(\mathbb{Q}\left(\zeta_{N}\right) / \mathbb{K}_{\mathcal{C}}\right) \rightarrow \operatorname{Gal}\left(\mathbb{Q}\left(\zeta_{N}\right)\right) \rightarrow \operatorname{Gal}(\mathcal{C}) \rightarrow 1,
$$

and it is known $([28])$ that $\operatorname{Gal}\left(\mathbb{Q}\left(\zeta_{N}\right) / \mathbb{K}_{\mathcal{C}}\right)$ is an elementary 2-group.

It follows from [6, Lem. 3.9] that if $d_{i}=\operatorname{FPdim}\left(X_{i}\right)$, for $i \in \operatorname{Irr}(\mathcal{C})$, then

$$
\hat{\sigma}\left(d_{i}\right)=d_{\hat{\sigma}(i)} \text {. }
$$

In particular, $\hat{\sigma}(0) \in G(\mathcal{C})$.

If $(s, t)$ is a normalized modular data for $\mathcal{C}$ then Galois Symmetry (cf. [12]) implies:

$$
\sigma^{2}\left(t_{i}\right)=t_{\hat{\sigma}(i)} \text { for all } i \text {. }
$$

2.7 Equivariantization of fusion categories. An action of the group $G$ on $\mathcal{C}$ is a monoidal functor $*: \underline{G} \rightarrow \operatorname{Aut}_{\otimes}(\mathcal{C})$. Here $\underline{G}$ is the discrete monoidal category whose simple objects are elements of $G$ and the tensor product is given by the product of $G$, and $\operatorname{Aut}_{\otimes}(\mathcal{C})$ is the monoidal category of tensor autoequivalences and tensor natural isomorphisms with tensor product given by composition.

Let $G$ be a group acting on $\mathcal{C}$ with action $*: \underline{G} \rightarrow \operatorname{Aut}_{\otimes}(\mathcal{C})$, so we have the following data

- tensor functors $\sigma_{*}: \mathcal{C} \rightarrow \mathcal{C}$, for each $\sigma \in G$,

- natural tensor isomorphisms $\phi(\sigma, \tau):(\sigma \tau)_{*} \rightarrow \sigma_{*} \circ \tau_{*}$, for all $\sigma, \tau \in G$,

- natural isomorphisms $\psi(\sigma)_{V, W}: \sigma_{*}(V) \otimes \sigma_{*}(W) \rightarrow \sigma_{*}(V \otimes W)$, for all $\sigma \in G$, $V, W \in \mathcal{C}$

Given an action $*: \underline{G} \rightarrow \operatorname{Aut}_{\otimes}(\mathcal{C})$ of $G$ on $\mathcal{C}$, the $G$-equivariantization of $\mathcal{C}$ is the fusion category $\mathcal{C}^{G}$ of $G$-equivariant objects defined as follows: An object in $\mathcal{C}^{G}$ is a pair $(V, f)$, where $V$ is an object of $\mathcal{C}$ and $f$ is a family of isomorphisms $f_{\sigma}: \sigma_{*}(V) \rightarrow V$, $\sigma \in G$, such that

$$
\phi(\sigma, \tau) f_{\sigma \tau}=f_{\sigma} \sigma_{*}\left(f_{\tau}\right)
$$

for all $\sigma, \tau \in G$. A $G$-equivariant morphism $\phi:(V, f) \rightarrow(W, g)$ between $G$-equivariant objects is a morphism $u: V \rightarrow W$ in $\mathcal{C}$ such that $g_{\sigma} \circ \sigma_{*}(u)=u \circ f_{\sigma}$, for all $\sigma \in G$.

The tensor product on $\mathcal{C}^{G}$ is defined by

$$
(V, f) \otimes(W, g):=(V \otimes W, h),
$$

where

$$
h_{\sigma}=f_{\sigma} g_{\sigma} \psi(\sigma)_{V, W}^{-1},
$$


and unit object $\left(\mathbf{1}, \mathrm{id}_{\mathbf{1}}\right)$.

2.8 De-equivariantization of fusion category We will denote by $\mathcal{Z}(\mathcal{D})$ the Drinfeld center of a fusion category $\mathcal{D}$, see $[20$ for its definition.

A central inclusion of $\operatorname{Rep}(G)$ in a fusion category $\mathcal{D}$ is a braided functor $\iota: \operatorname{Rep}(G) \hookrightarrow$ $\mathcal{Z}(\mathcal{D})$ such that the composition $\operatorname{Rep}(G) \hookrightarrow \mathcal{Z}(\mathcal{D}) \rightarrow \mathcal{D}$ is an inclusion.

Let $\iota: \operatorname{Rep}(G) \hookrightarrow \mathcal{Z}(\mathcal{D})$ be a central inclusion. The algebra $\mathbb{C}^{G}:=\{f: G \rightarrow \mathbb{C}\}$ is a commutative algebra in $\operatorname{Rep}(G)$, so it is a commutative algebra in $\mathcal{Z}(\mathcal{D})$. Thus the category $\mathcal{C}_{G}$ of right $\mathbb{C}^{G}$-modules in $\mathcal{D}$, called the de-equivariantization, is a fusion category with tensor product $\otimes_{\mathbb{C}^{G}}$ and unit $\mathbb{C}^{G}$.

If $\mathcal{C}^{G}$ is a $G$-equivariantization then there is a canonical inclusion $\iota: \operatorname{Rep}(G) \hookrightarrow \mathcal{C}^{G}$, that lifts to a central inclusion $\iota: \operatorname{Rep}(G) \hookrightarrow \mathcal{Z}\left(\mathcal{C}^{G}\right)$ and $\left(\mathcal{C}^{G}\right)_{G} \cong_{\otimes} \mathcal{C}$. Conversely, if $\mathcal{D}_{G}$ is a de-equivariantization, $G$ acts on $\mathcal{D}_{G}$ and $\left(\mathcal{D}_{G}\right)^{G} \cong \mathcal{D}$, 13.

If $\mathcal{C}$ is a braided fusion category every Tannakian subcategory $\operatorname{Rep}(G) \subseteq \mathcal{C}$ has a canonical central inclusion structure so there is an associated de-equivariantization $\mathcal{C}_{G}$.

For a complete treatment of (de-)equivariantization of fusion categories see [13].

\section{$3 \operatorname{dim} \mathcal{C}=4 m, m$ ODD SQUARE-FREE INTEGER}

Using [17, Prop. 8.23] we may and will assume that all weakly integral modular categories in the following have the canonical positive spherical structure, with respect to which categorical dimensions of simple objects coincide with their Frobenius-Perron dimensions.

Theorem 3.1. Suppose that $\mathcal{C}$ is a modular category with $\operatorname{dim} \mathcal{C}=4 m$, with $m$ an odd square-free integer. Then exactly one of the following is true:

(a) $\mathcal{C}$ contains an object of dimension $\sqrt{2}$ and $\mathcal{C}$ is equivalent to a Deligne product of an Ising modular category and a cyclic modular category or

(b) $\mathcal{C}$ is non-integral and contains no objects of dimension $\sqrt{2}$ and $\mathcal{C}$ is equivalent to a Deligne product of a $\mathbb{Z}_{2}$-equivariantization of a Tambara-Yamagami category over $\mathbb{Z}_{k}$ and a cyclic modular category of dimension $n$ where $1 \leq n=\frac{m}{k} \in \mathbb{Z}$ or

(c) $\mathcal{C}$ is pointed.

All equivalences are as premodular fusion categories. 
Proof. If $d_{i} \in \mathbb{Z}$ then $d_{i}=1$ or 2 since $d_{i}^{2} \mid 4 m$ (by [15, Lemma 1.2], [17, Proposition 8.27], [16, Proposition 2.11(i)]).

First, we claim that if $4 \mid \operatorname{dim} \mathcal{C}_{p t}$ then $\mathcal{C}$ is pointed. Under this assumption, with respect to the universal $U(\mathcal{C})$-grading, each component has odd dimension. In particular, $\operatorname{dim} \mathcal{C}_{a d}$ is odd, so $\mathcal{C}_{a d} \subset \mathcal{C}_{\text {int }}$ cannot contain simple objects of even dimension. Therefore, $\mathcal{C}_{a d}$ is pointed and hence $\mathcal{C}$ is nilpotent. By [13, Thm. 1.1], $\mathcal{C}$ has a unique decomposition into a tensor product of braided fusion categories whose Frobenius-Perron dimensions are the distinct prime power factors of $\operatorname{dim} \mathcal{C}=4 m$. Since braided fusion categories with prime Frobenius-Perron dimensions are pointed and 4||$G(\mathcal{C}) \mid$, all the factors in this tensor decomposition of $\mathcal{C}$ are pointed. Thus, $\mathcal{C}$ is pointed.

If $\mathcal{C}$ is integral, then $4 m=\operatorname{dim} \mathcal{C}_{p t}+4 s$, where $s=\left|\left\{i \in \operatorname{Irr}(\mathcal{C}) \mid d_{i}=2\right\}\right|$. Therefore, $4 \mid \operatorname{dim} \mathcal{C}_{p t}$ and hence, by the previous paragraph, $\mathcal{C}$ is pointed as described in (c).

We may therefore assume that $4 \nmid \operatorname{dim} \mathcal{C}_{p t}$ and $d_{i} \notin \mathbb{Z}$ for some $i \in \operatorname{Irr}(\mathcal{C})$. Then $|U(\mathcal{C})|=\operatorname{dim} \mathcal{C}_{p t}=2^{a} n$, where $a=0,1$ and $n \mid m$ is odd and square-free. By 19 , Theorem 3.10] $a \neq 0$, so we must have $a=1$.

We claim that $\mathcal{C} \cong \mathcal{D} \otimes \mathcal{P}$, where $\mathcal{D}$ is modular with $\operatorname{dim} \mathcal{D}=4 k$ and $\operatorname{dim} \mathcal{D}_{p t}=2$ and $\mathcal{P}$ is a cyclic modular category of dimension $n=m / k$. We prove this by induction on the number of (distinct) primes factors of $n$. In the base case $n=1$ ( 0 prime factors) the statement is clear, since $\mathcal{C} \cong \mathcal{C} \otimes$ Vec. Suppose $p$ is a prime factor of $n$. Let $\mathcal{C}_{p t}(p)$ be a pointed subcategory of dimension $p$. Observe that $\mathcal{C}_{p t}(p)$ is either symmetric or modular. If $\mathcal{C}_{p t}(p) \cong \operatorname{Rep}\left(\mathbb{Z}_{p}\right)$ is symmetric then it is also Tannakian since $p$ is odd (cf. [14, Corollary 2.50 (i)]). The corresponding $\mathbb{Z}_{p}$-de-equivariantization of $\mathcal{C}$ is $\mathbb{Z}_{p}$-graded with trivial component $\left(\mathcal{C}_{\mathbb{Z}_{p}}\right)_{0}$ of dimension $\operatorname{dim} \mathcal{C} / p^{2}$, contradicting $n$ squarefree. Therefore $\mathcal{C}_{p t}(p)$ is modular and hence $\mathcal{C} \cong \mathcal{C}_{p t}(p) \otimes \mathcal{D}$ where $\mathcal{D}$ is the modular subcategory which centralizes $\mathcal{C}_{p t}(p)$ by [23, Theorem 4.2] (see also [14, Theorem 3.13]). Now $|U(\mathcal{D})|=2 n / p$ so the claim follows.

We now proceed to classify modular categories $\mathcal{D}$ with $\operatorname{dim} \mathcal{D}=4 k$ and $\operatorname{dim} \mathcal{D}_{p t}=2$. If $k=1$, then $\operatorname{dim} \mathcal{D}=4$ and $\mathcal{D}$ is an Ising modular category by [14, Appendix B]. In this case, $\mathcal{C}$ is as in (a).

Now, we may assume $k>1$. Since $U(\mathcal{D}) \cong \mathbb{Z}_{2}, \mathcal{D}$ has a $\mathbb{Z}_{2}$-grading $\mathcal{D}=\mathcal{D}_{0} \oplus \mathcal{D}_{1}$ where $\mathcal{D}_{0}=\mathcal{D}_{a d}$ is the trivial component. For any simple object $X_{i}$ with $d_{i} \notin \mathbb{Z}, X_{i} \in \mathcal{D}_{1}$ and $d_{i}=\alpha_{i} \sqrt{\ell}$, with $\ell$ square-free and $\alpha_{i} \in\{1,2\}$, since $\mathcal{D}$ is modular and $k$ is square free. The trivial component $\mathcal{D}_{0}$ contains all the simple objects of integral dimension, which consist of two isomorphism classes of dimension 1 and $(k-1) / 2$ classes of dimension 2 . 
Let $\sigma \in \operatorname{Gal}(\mathcal{D})$ which assigns $\sqrt{\ell} \mapsto-\sqrt{\ell}$ and $\hat{\sigma}(0)=1$. Then the second row of the $S$-matrix of $\mathcal{D}$ is given by the action $\sigma$ on the first row of $S$. Therefore, the second row of $S$ (corresponding to label $1 \in \operatorname{Irr}(\mathcal{C})$ ) is:

$$
\left(1,1,2, \ldots, 2,-\alpha_{1} \sqrt{\ell}, \ldots,-\alpha_{t} \sqrt{\ell}\right) .
$$

In particular, $1 \in \operatorname{Irr}(\mathcal{C})$ must be the isomorphism class of a non-trivial invertible object $g$. We claim that $\theta_{g}=1$. To see this, first observe that if $Y$ is simple and $\operatorname{dim}(Y)=2$ then $Y \otimes Y^{*}=\mathbf{1} \oplus g \oplus Y^{\prime}$, where $Y^{\prime}$ is a 2-dimensional simple object. Thus, $N_{Y, Y^{*}}^{g}=N_{Y, g}^{Y}=1$ so that $Y \otimes g \cong Y$, for all 2-dimensional simple object $Y$. The balancing equation 2.2 gives:

$$
2=S_{g, Y}=\theta_{g}^{-1} \theta_{Y}^{-1} N_{g^{*}, Y}^{Y} d_{Y} \theta_{Y}=2 \theta_{g}^{-1},
$$

so that $\theta_{g}=1$, as claimed.

For $i \in \operatorname{Irr}\left(\mathcal{D}_{1}\right), d_{i}=\alpha_{i} \sqrt{\ell}$. We use the balancing equation $(2.2)$ again:

$$
-\alpha_{i} \sqrt{\ell}=S_{g, X_{i}}=\theta_{g}^{-1} \theta_{X_{i}}^{-1} \theta_{g \otimes X_{i}} d_{g \otimes X_{i}}=\alpha_{i} \sqrt{\ell} \theta_{X_{i}}^{-1} \theta_{g \otimes X_{i}}
$$

which implies that $\theta_{X_{i}}=-\theta_{g \otimes X_{i}}$. Consequently, $g \otimes X_{i} \neq X_{i}$ and $g \otimes X_{i} \neq X_{i}^{*}$ so that $N_{X_{i}, X_{i}}^{g}=N_{X_{i}, X_{i}^{*}}^{g}=0$. This implies $X_{i} \otimes X_{i}^{*} \cong \mathbf{1} \oplus \sum_{j} m_{j} Y_{j}$, where $Y_{j}$ are simple objects of dimension 2. In particular, $d_{i}^{2}=\alpha_{i}^{2} \ell$ is odd. Thus $\ell$ is odd and we conclude $\alpha_{i}=1$, for all $i \in \operatorname{Irr}\left(\mathcal{D}_{1}\right)$. Notice that, since $d_{i}^{2}=\ell$ is odd and $N_{X_{i}, X_{i}}^{g}=0$, we must have $X_{i} \otimes X_{i} \cong \mathbf{1} \oplus \sum_{j} n_{j} Y_{j}$ with $\operatorname{FPdim}\left(Y_{j}\right)=2$ so that $X_{i}$ is self-dual.

We claim that $\left|\operatorname{Irr}\left(\mathcal{D}_{1}\right)\right|=2$. Since $g$ does not fix any $X_{i},\left|\operatorname{Irr}\left(\mathcal{D}_{1}\right)\right|>1$. Let $j \in \operatorname{Irr}\left(\mathcal{D}_{1}\right)$. Since $\operatorname{dim}\left(X_{i} \otimes X_{j}\right)=\ell$ is odd, exactly one of 1 or $g$ is a subobject of $X_{i} \otimes X_{j}$. Since all $X_{i}$ are self-dual, $N_{X_{i}, X_{j}}^{1}=1$ implies $X_{i} \cong X_{j}$ whereas $N_{X_{i}, X_{j}}^{g}=1$ implies $X_{j} \cong g \otimes X_{i}$. Therefore, $X_{i}$ and $g \otimes X_{i} \neq X_{i}$ represent the only two non-isomorphic simple objects in $\mathcal{D}_{1}$. Thus, we conclude that $\ell=k$, and $|\operatorname{Irr}(\mathcal{D})|=(k+7) / 2$. Let $\left\{Y_{2}, \ldots, Y_{(k+1) / 2}\right\}$ be a complete set of representatives for the simple objects of dimension 2 , and $\{U, V\} \subset \mathcal{D}_{1}$ for dimension $\sqrt{k}$. Since $\theta_{g}=1$, the subcategory generated by $g$ is Tannakian. This induces an action of $G(\mathcal{D})$ on $\mathcal{D}$ by interchanging 1 and $g$, fixing all $Y_{i}$ and interchanging $U$ and $V$. Then the $\mathbb{Z}_{2}$-de-equivariantization $\mathcal{D}_{\mathbb{Z}_{2}}$ has $k$ objects of dimension 1 (one from the $G(\mathcal{C})$-orbit $\{\mathbf{1}, g\}$ and two from each $Y_{i}$ ) and one object of dimension $\sqrt{k}$ (from the orbit $\{U, V\}$ ) (cf. [22, 5.1]). As a fusion category $\mathcal{D}_{\mathbb{Z}_{2}}$ must be $T Y\left(\mathbb{Z}_{k}, \chi, \nu\right)$, for which there are 4 possible choices of $\chi$ and $\nu$. Since equivariantization and de-equivariantization are inverse operations, we have $\mathcal{D} \cong T Y\left(\mathbb{Z}_{k}, \chi, \nu\right)^{\mathbb{Z}_{2}}$. As these categories are described in (b), the proof is complete.

Remark 3.2. An alternative construction of the categories obtained in Theorem 3.1 (b) is as follows. The non-pointed factor $\mathcal{D}:=T Y\left(\mathbb{Z}_{k}, \chi, \nu\right)^{\mathbb{Z}_{2}}$ can be recovered from 
the formula

$$
\mathcal{D} \otimes \mathcal{P} \cong \mathcal{Z}\left(T Y\left(\mathbb{Z}_{k}, \chi, \nu\right)\right)
$$

obtained from [10, Cor. 3.30]. Here $\mathcal{P}$ is the maximal pointed subcategory of the Drinfeld center $\mathcal{Z}\left(T Y\left(\mathbb{Z}_{k}, \chi, \nu\right)\right)$, which is modular by $[18$, Prop. 4.2]. There are at most 8 possible inequivalent categories of this form up to balanced braided tensor equivalences: 4 for the choices of $\chi$ and $\nu$ (two each) and two choices of a spherical structure.

The following result will be useful later. We are indebted to an anonymous referee whose comment inspired a strengthening of this result. Although we only need this result in the weakly integral case, the general case is of independent interest. A Fibonacci category is a rank 2 modular category of dimension $\frac{5+\sqrt{5}}{2}$.

Lemma 3.3. If $\mathcal{C}$ is a modular category in which there is a unique simple isomorphism class of objects $X$ such that $\mathrm{FP} \operatorname{dim} X \notin \mathbb{Z}$ then $\mathcal{C}$ is either equivalent to an Ising modular category or to a Fibonacci category.

Proof. We first claim that under these hypotheses $\mathcal{C}$ is either a Fibonacci category or (strictly) weakly integral.

Let $Y$ be any simple object of integer dimension. Since FPdim $X \notin \mathbb{Z}$ and is the only such simple object we see that $Y \otimes X \cong \mathrm{FPdim}(Y) X$ by comparing dimensions. Setting $x=\mathrm{FPdim}(X)$ we see that $x$ satisfies the quadratic relation: $x^{2}-k x-\ell=0$ with $k, \ell \in \mathbb{Z}$. Let $u$ be the other root of this equation and $\sigma$ the corresponding Galois automorphism of $\mathbb{Q}(x)$ with $\sigma(x)=u$. Since the first column $C_{0}$ of the $S$-matrix has exactly one irrational entry, $\sigma\left(C_{0}\right)=C_{i} / \mathrm{FPdim}\left(Y_{i}\right)$ for some simple object $Y_{i}$. Since $C_{0}$ and $C_{i}$ have the same lengths, we obtain the equation

$$
\operatorname{FPdim}\left(\mathcal{C}_{\text {int }}\right)+x^{2}=\operatorname{FPdim}\left(Y_{i}\right)^{2}\left(\operatorname{FPdim}\left(\mathcal{C}_{\text {int }}\right)+u^{2}\right)
$$

Suppose first that $Y_{i} \neq X$, i.e. $\operatorname{FPdim}\left(Y_{i}\right) \in \mathbb{Z}$. Since $\operatorname{FPdim}\left(\mathcal{C}_{\text {int }}\right), x^{2}+u^{2} \in \mathbb{Z}$ we see that $x^{2} \in \mathbb{Z}$ and hence $\mathcal{C}$ is weakly integral.

Alternatively, FPdim $\left(Y_{i}\right)=x$ from which we obtain $\left(\right.$ FPdim $\left.\mathcal{C}_{\text {int }}-1\right) x^{2} \in \mathbb{Z}$ which implies either $x^{2} \in \mathbb{Z}$ (weakly integral) or FPdim $\mathcal{C}_{i n t}=1$. Since the Fibonacci categories are the only non-integral rank 2 modular categories we have established the first claim.

Thus we may assume that FPdim $X=\sqrt{m} \notin \mathbb{Z}$. Observe that $X \otimes X$ must be a sum of simple objects of integral dimension and as above we have $Y \otimes X \cong \operatorname{FPdim}(Y) X$. Thus, by $[19]$, the universal grading group is $U(\mathcal{C}) \cong \mathbb{Z}_{2}$ from which we conclude that there is a (unique up to isomorphism) invertible object $g$, and FPdim $\mathcal{C}=2 m$. Both $X$ and the non-trivial invertible object $g$ are self-dual. The first column of the $S$-matrix 
is given by the Frobenius-Perron dimensions of the simple objects of $\mathcal{C}$. Let the index $1 \in \operatorname{Irr}(\mathcal{C})$ correspond to the non-trivial invertible object $g$. By [6, Lemma 3.9], the Galois automorphism $\sigma$ that sends $\sqrt{m}$ to $-\sqrt{m}$ interchanges the first two columns of the $S$-matrix, so that they differ only is the last entries. Since each column of $S$ has the same length (i.e. $\operatorname{dim} \mathcal{C}=2 m$ ), we can conclude that only the first two entries of the last column are non-zero. Thus the $S$-matrix of $\mathcal{C}$ is:

$$
\left[\begin{array}{cccccc}
1 & 1 & d_{1} & \cdots & d_{t} & \sqrt{m} \\
1 & 1 & d_{1} & \cdots & d_{t} & -\sqrt{m} \\
d_{1} & d_{1} & & & & 0 \\
\vdots & \vdots & & \ddots & & \vdots \\
d_{t} & d_{t} & & & & 0 \\
\sqrt{m} & -\sqrt{m} & 0 & \cdots & 0 & 0
\end{array}\right]
$$

where $1<d_{i} \in \mathbb{Z}$. Applying the twist equation (2.1) to the entry $S_{X, X}=0$, we get that $0=\theta_{X}^{2} \sum_{i} \theta_{i} S_{i, X}^{2}=\left(m \theta_{\mathbf{1}}+m \theta_{g}\right) \theta_{X}^{2}$. We conclude that $\theta_{g}=-\theta_{\mathbf{1}}=-1$. Next, we apply the twist equation (2.1) to the entry $S_{g, X}=-\sqrt{m}$ obtaining:

$$
p_{+}(-\sqrt{m})=\theta_{g} \theta_{X} \sum_{i} \theta_{i} S_{i, g} S_{i, X}=-\theta_{X} 2 \sqrt{m} .
$$

It follows that $\left|p_{+}\right|=2\left|\theta_{X}\right|=2$ and $\operatorname{dim} \mathcal{C}=4$. Therefore $\mathcal{C}$ is equivalent to an Ising modular category.

Lemma 3.4. Let $\mathcal{C}$ be a weakly integral modular category of square-free FrobeniusPerron dimension. Then $\mathcal{C}$ is pointed.

Proof. By [15], the only possible integral FP-dimension of a simple object is 1 , since $\operatorname{FPdim}(\mathcal{C})$ is square-free. Therefore, the integral subcategory $\mathcal{C}_{a d}$ is pointed, hence $\mathcal{C}$ is nilpotent. Thus, by [14, Theorem 1.1], $\mathcal{C}$ is a Deligne product of braided subcategories of prime dimension. Such categories are pointed, by [17, Corollary 8.30].

\section{General Technical Results}

The results of the first two subsections apply to arbitrary modular categories. In particular, we do not assume that $d_{i}=\mathrm{FPdim}\left(X_{i}\right)$, since this cannot always be achieved (even by changing the spherical structure). We introduce an action of $G(\mathcal{C})$ on $\operatorname{Irr}(\mathcal{C})$ and study its restriction to symmetric pointed subcategories. Then we explore the cycle structure of the permutation action of $\operatorname{Gal}(\mathcal{C})$ on $\operatorname{Irr}(\mathcal{C})$, particularly for elements coming from automorphisms of $\mathbb{Q}_{p^{a}}$ for primes $p$ dividing $N=\operatorname{FSexp}(\mathcal{C})$.

The third subsection studies support cycles for weakly integral modular categories specifically, to be applied in our classification. 
4.1 $G(\mathcal{C})$-grading of a modular category Let $G=G(\mathcal{C})$ be the group of isomorphism classes of invertible objects of a modular category $\mathcal{C}$. The abelian group $G$ acts on $\operatorname{Irr}(\mathcal{C})$ as follows: Let $i \in G$ and $j \in \operatorname{Irr}(\mathcal{C})$ and $X_{i}, X_{j}$ be representative simple objects in these classes. Since $X_{i}$ is an invertible object, $X_{i}^{*} \otimes X_{j} \cong X_{k}$ is simple and we define $i \cdot j=k$.

Let $\hat{G}$ denote the character group of $G$. For each $j \in \operatorname{Irr}(\mathcal{C})$, the map $\phi_{j}: i \mapsto S_{i j} / d_{j}$ defines a character of the Grothendieck ring $K_{0}(\mathcal{C})$. Notice that modularity implies that the restrictions of the $\phi_{j}$ for all $j \in \operatorname{Irr}(\mathcal{C})$ span $\hat{G}$. Since for $i \in G, d_{i}=1 / d_{i}= \pm 1$, the set of characters $\phi_{0} \phi_{j}: i \mapsto \frac{S_{i j}}{d_{i} d_{j}}$ for all $j \in \operatorname{Irr}(\mathcal{C})$ also spans $\hat{G}$. Thus, the modular category $\mathcal{C}$ admits a faithful $\hat{G}$-grading (cf. 19 , Theorem 6.2]) which is given by $\mathcal{C}=\bigoplus_{\chi \in \hat{G}} \mathcal{C}_{\chi}$, where the set of simple objects in $\mathcal{C}_{\chi}$ are:

$$
\operatorname{Irr}\left(\mathcal{C}_{\chi}\right)=\left\{j \in \operatorname{Irr}(\mathcal{C}) \mid \frac{S_{i j}}{d_{i} d_{j}}=\chi(i), \text { for all } i \in G\right\} .
$$

This natural $\hat{G}$-grading on $\mathcal{C}$ induces a canonical $\hat{H}$-grading on $\mathcal{C}$, for any subgroup $H$ of $G$. More precisely, for $\chi \in \hat{H}$, the set of simple objects in each component of the grading is:

$$
\operatorname{Irr}\left(\mathcal{C}_{\chi}\right)=\left\{j \in \operatorname{Irr}(\mathcal{C}) \mid \frac{S_{i j}}{d_{i} d_{j}}=\chi(i), \text { for all } i \in H\right\} .
$$

Since the restriction map $\hat{G} \rightarrow \hat{H}$ is surjective, this $\hat{H}$-grading is also faithful and $\operatorname{dim} \mathcal{C}_{\chi}=\frac{\operatorname{dim} \mathcal{C}}{|H|}$, for all $\chi \in \hat{H}$. See $[17$, Proposition 8.20].

The subcategory generated by a subgroup $H \subset G$ will be denoted $\mathcal{C}(H)$. For example $\mathcal{C}(G)=\mathcal{C}_{p t}$. We are particular interested in the subgroups $H$ of $G$ which generates a symmetric full subcategory of $\mathcal{C}$. In this case, we will simply call $H$ a self-centralizing subgroup of $G$.

Remark 4.1. If $H$ is a self-centralizing subgroup of $G$ then $S_{g, h}=d_{g} d_{h}$, for all $g, h \in H$. Therefore, $H \subset \mathcal{C}_{e}$, where $\mathcal{C}_{e}$ is the trivial component of the $\hat{H}$-grading of $\mathcal{C}$ associated to the trivial character. Moreover if $g, h \in H$ we have

$$
\left(d_{h} \theta_{h}\right)\left(d_{g} \theta_{g}\right)=\left(d_{h^{*}} \theta_{h^{*}}\right)\left(d_{g} \theta_{g}\right)=d_{h g} \theta_{h g}
$$

by (2.2) since $S_{g^{*}, h}=d_{g^{*}} d_{h}$. Therefore $\chi_{H}(h)=d_{h} \theta_{h}$ defines a character of $H$. If $\mathcal{C}(H)$ is a Tannakian subcategory of $\mathcal{C}$ then $H$ is a self-centralizing subgroup and $d_{h}=\theta_{h}= \pm 1$, for all $h \in H$, so $\chi_{H}$ is trivial. We will simply call such subgroups Tannakian. Notice that if $H$ is a self-centralizing group of odd prime order $p$ then $H$ is Tannakian by 14 , Corollary 2.50]. 
On the other hand, if the self-centralizing subgroup $H$ is not Tannakian, then there exists $h \in H$ such that $d_{h} \theta_{h}=-1$ so that $\chi_{H}$ is a non-trivial character. This condition provides a faithful $\mathbb{Z}_{2}$-grading on the fusion subcategory $\mathcal{C}(H)$ generated by $H$. The set of isomorphism classes $\operatorname{Irr}\left(\mathcal{C}(H)_{0}\right)$ of simple objects of the trivial component $\mathcal{C}(H)_{0}$ is an index 2 Tannakian subgroup. In particular, $H$ must be of even order.

Lemma 4.2. Let $H$ be a self-centralizing subgroup of $G$ and $\chi \in \hat{H}$. Then $H \cdot j \subseteq$ $\operatorname{Irr}\left(\mathcal{C}_{\chi}\right)$, for all $j \in \operatorname{Irr}\left(\mathcal{C}_{\chi}\right)$.

(i) If $H$ is Tannakian and $\chi$ is not trivial then $H \cdot j$ is not a singleton for any $j \in \operatorname{Irr}\left(\mathcal{C}_{\chi}\right)$. In particular, when $H$ is Tannakian of prime order and $\chi$ is not trivial, $|H|$ divides $\left|\operatorname{Irr}\left(\mathcal{C}_{\chi}\right)\right|$.

(ii) Suppose $H$ is not Tannakian and $\chi \in \hat{H}$. If some $j \in \operatorname{Irr}\left(\mathcal{C}_{\chi}\right)$ satisfies $H \cdot j=$ $\{j\}$ then $\chi=\chi_{H}$.

Proof. Since $H \subseteq \mathcal{C}_{e}$ and the action of $H$ is induced by the tensor product, it follows immediately from the definition of grading that $H \cdot \operatorname{Irr}\left(\mathcal{C}_{\chi}\right) \subseteq \operatorname{Irr}\left(\mathcal{C}_{\chi}\right)$.

Now, we assume that $H$ generates a Tannakian subcategory of $\mathcal{C}$. Thus $d_{h} \theta_{h}=1$, for all $h \in H$. Suppose that $j \in \operatorname{Irr}\left(\mathcal{C}_{\chi}\right)$ is fixed by $H$. Then $N_{h, j}^{k}=\delta_{k, j}$, for all $h \in H$, $k \in \operatorname{Irr}(\mathcal{C})$. Using the balancing equation $(2.2)$, we get that

$$
\chi(h)=\frac{S_{h, j}}{d_{h} d_{j}}=\frac{\sum_{k} N_{h^{*}, j}^{k} d_{k} \theta_{k}}{\theta_{h} \theta_{j} d_{h} d_{j}}=1,
$$

for all $h \in H$. Therefore, $\chi$ is trivial. In addition, $|H|=p$ implies that each $H$-orbit in $\operatorname{Irr}\left(\mathcal{C}_{\chi}\right)$ has exactly $p$ classes. Therefore, the second statement of (i) follows.

Suppose $H$ is not Tannakian, and $j \in \operatorname{Irr}\left(\mathcal{C}_{\chi}\right)$ for some $\chi \in \hat{H}$ satisfies $H \cdot j=\{j\}$. Then, for $h \in H$, the balancing equation (2.2) implies

$$
\chi(h)=\frac{S_{h j} \theta_{h} \theta_{j}}{d_{h} d_{j} \theta_{h} \theta_{j}}=\frac{d_{h \cdot j} \theta_{h \cdot j}}{d_{h} d_{j} \theta_{h} \theta_{j}}=\frac{\theta_{j} d_{j}}{d_{h} d_{j} \theta_{h} \theta_{j}}=\frac{1}{d_{h} \theta_{h}}=\chi_{H}(h),
$$

since $\theta_{h}= \pm 1$ for $h \in H$.

Lemma 4.3. Suppose $H$ is a self-centralizing subgroup of $G$. If, for some $\psi \in \hat{H}$, $\operatorname{Irr}\left(\mathcal{C}_{\psi}\right)$ consists of only one $H$-orbit then $\mathcal{C}_{e}$ is integral. If, in addition, $k \in \operatorname{Irr}\left(\mathcal{C}_{e}\right)$ is fixed by $H$ then $\left[H: H_{0}\right] \mid d_{k}$, where $H_{0}$ is the stabilizer of any element of $\operatorname{Irr}\left(\mathcal{C}_{\psi}\right)$.

Proof. Since $H$ is self-centralizing then $H \subseteq \mathcal{C}_{e}$, see Remark 4.1. Let $R=\sum_{i \in \operatorname{Irr}(\mathcal{C})} d_{i} X_{i} \in$ $K_{0}(\mathcal{C}) \otimes \mathbb{R}$ be the virtual regular object of $\mathcal{C}$. Then $R=\sum_{\chi \in \hat{H}} R_{\chi}$, where $R_{\chi}=$ $\sum_{i \in \operatorname{Irr}\left(\mathcal{C}_{\chi}\right)} d_{i} X_{i}$ is the regular object of the component $\mathcal{C}_{\chi}$. 
Suppose $\operatorname{Irr}\left(\mathcal{C}_{\psi}\right)=H \cdot j$, with $j \in \operatorname{Irr}\left(\mathcal{C}_{\psi}\right)$. Let $H_{0}$ be the stabilizer of the $H$-orbit $\operatorname{Irr}\left(\mathcal{C}_{\psi}\right)$. Note that the quotient group $\bar{H}=H / H_{0}$ acts on $j$ as $\bar{h} \cdot j=h \cdot j$, where $\bar{h}=h H_{0}$. Since $d_{j}=d_{h \cdot j}=d_{h} d_{j}$ for $h \in H_{0}$, we have $d_{h}=1$ for $h \in H_{0}$. Therefore, we can define $d_{\bar{h}}=d_{h}$ for all $h \in H$. Thus $R_{\psi}=d_{j} \sum_{\bar{h} \in \bar{H}} d_{\bar{h}} X_{\bar{h} \cdot j}=\frac{d_{j}}{\left|H_{0}\right|} \sum_{h \in H} d_{h} X_{h}^{*} \otimes X_{j}$. Since each $\mathcal{C}_{\chi}$ is a $\mathcal{C}_{e}$-module category, and $X_{k} \otimes R=d_{k} R$, for $k \in \operatorname{Irr}\left(\mathcal{C}_{e}\right)$, we have $X_{k} \otimes R_{\chi}=d_{k} R_{\chi}$, for all $\chi \in \hat{H}$. On the other hand,

$$
\begin{aligned}
X_{k} \otimes R_{\psi}=d_{j} \sum_{\bar{h} \in \bar{H}} d_{\bar{h}} X_{h}^{*} \otimes X_{j} \otimes X_{k}=d_{j} \sum_{\bar{h}, \overline{h^{\prime}} \in \bar{H}} d_{\bar{h}} n_{\overline{h^{\prime}}} X_{h}^{*} \otimes X_{\overline{h^{\prime}} \cdot j} \\
\quad=d_{j} \sum_{\bar{h}, \overline{h^{\prime}} \in \bar{H}} d_{\bar{h}} n_{\overline{h^{\prime}}} X_{\overline{h h^{\prime} \cdot j}}=d_{j} \sum_{h, h^{\prime} \in \bar{H}} d_{\overline{h^{\prime}}} n_{\bar{h} \overline{h^{\prime-1}}} X_{\bar{h} \cdot j}=n R_{\psi},
\end{aligned}
$$

where $n=\sum_{\bar{h} \in \bar{H}} d_{\bar{h}} n_{\bar{h}}$ is an integer. Therefore, $d_{k}=n \in \mathbb{Z}$ and $\mathcal{C}_{e}$ is integral, as claimed.

If $k$ is fixed by $H$ then $d_{h}=1$ for all $h \in H$ and

$d_{k} R_{\psi}=X_{k} \otimes R_{\psi}=\frac{d_{j}}{\left|H_{0}\right|} \sum_{h \in H} X_{k} \otimes d_{h} X_{h}^{*} \otimes X_{j}=d_{j} \frac{|H|}{\left|H_{0}\right|} X_{j} \otimes X_{k}=d_{j} \frac{|H|}{\left|H_{0}\right|} \sum_{\overline{h^{\prime}} \in \bar{H}} n_{\overline{h^{\prime}}} X_{\overline{h^{\prime}} \cdot j}$

Thus, $\frac{|H|}{\left|H_{0}\right|} n_{\bar{h}}=d_{k}$, for all $\bar{h} \in \bar{H}$. In particular, $\frac{|H|}{\left|H_{0}\right|} \mid d_{k}$ and $n_{\bar{h}}=n_{\overline{h^{\prime}}}$, for $\bar{h}, \overline{h^{\prime}} \in \bar{H}$.

Since $\chi \in \hat{H}$ is of the form $\frac{S_{h, j}}{d_{h} d_{j}}$ for some $j \in \operatorname{Irr}(\mathcal{C})$, for each $\sigma \in \operatorname{Gal}(\mathcal{C})$ we may define $(\sigma \circ \chi)(h)=\frac{\sigma\left(S_{h, j}\right)}{\sigma\left(d_{h}\right) \sigma\left(d_{j}\right)}$. In the following we explore the relationship between the actions of $\operatorname{Gal}(\mathcal{C})$ and $G(\mathcal{C})$.

Lemma 4.4. Let $\mathcal{C}$ be a modular category and $H$ a subgroup of $G(\mathcal{C})$.

(i) Suppose that $j \in \operatorname{Irr}\left(\mathcal{C}_{\chi}\right)$ for some $\chi \in \hat{H}$. Then, for $\sigma \in \operatorname{Gal}(\mathcal{C})$ we have $\hat{\sigma}(j) \in \mathcal{C}_{\sigma \circ \chi}$. In particular, if $\operatorname{ord}(\chi)=1$ or $2, \hat{\sigma}\left(\operatorname{Irr}\left(\mathcal{C}_{\chi}\right)\right)=\operatorname{Irr}\left(\mathcal{C}_{\chi}\right)$.

(ii) If $H$ is self-centralizing elementary 2-group, then the actions of $H$ and $\operatorname{Gal}(\mathcal{C})$ on $\operatorname{Irr}(\mathcal{C})$ commute.

Proof. (i) For $j \in \operatorname{Irr}\left(\mathcal{C}_{\chi}\right), \sigma\left(\frac{S_{i j}}{d_{j}}\right)=\frac{S_{i \hat{\imath}(j)}}{d_{\hat{\sigma}(j)}}$ for all $i \in \operatorname{Irr}(\mathcal{C})$. Thus, for $h \in H$, we have

$$
\sigma(\chi(h))=\sigma\left(\frac{S_{h j}}{d_{h} d_{j}}\right)=\frac{S_{h \hat{\sigma}(j)}}{d_{h} d_{\hat{\sigma}(j)}} .
$$

Therefore, $\hat{\sigma}(j) \in \operatorname{Irr}\left(\mathcal{C}_{\sigma \circ \chi}\right)$. If $\operatorname{ord}(\chi) \mid 2$, then $\chi(h)= \pm 1$ for all $h \in H$. Hence, $\sigma \circ \chi=\chi$ for all $\sigma \in \operatorname{Gal}(\mathcal{C})$. The second statement of (i) follows. 
(ii) Suppose $H$ is self-centralizing elementary 2-group, and let $\chi, \psi \in \hat{H}$. For $h \in H$, $j \in \operatorname{Irr}\left(\mathcal{C}_{\chi}\right)$ and $i \in \operatorname{Irr}\left(\mathcal{C}_{\psi}\right)$ we have $X_{i}^{*} \otimes X_{j} \in \mathcal{C}_{\chi \psi}$ (since $H$ a 2-group implies $\left.X_{i}^{*} \in \operatorname{Irr}\left(\mathcal{C}_{\psi}\right)\right)$ and

Thus,

$$
\chi(h)=\frac{S_{h, j}}{d_{h} d_{j}}=\frac{\theta_{h \cdot j}}{\theta_{h} \theta_{j}} .
$$

Therefore,

$$
\begin{aligned}
\frac{S_{i, h \cdot j}}{d_{h \cdot j}}=\frac{\sum_{k} N_{i^{*}, j}^{k} d_{h \cdot k} \theta_{h \cdot k}}{\theta_{i} \theta_{h \cdot j} d_{h} d_{j}} & =\frac{\sum_{k} N_{i^{*}, j}^{k} d_{k} \chi \psi(h) \theta_{h} \theta_{k}}{\theta_{i} \chi(h) \theta_{h} \theta_{j} d_{j}} \\
& =\frac{\sum_{k} N_{i^{*}, j}^{k} d_{k} \theta_{k} \psi(h)}{\theta_{i} \theta_{j} d_{j}}=\frac{S_{i, j} \psi(h)}{d_{j}} .
\end{aligned}
$$

$$
\frac{S_{i, h \cdot \hat{\sigma}(j)}}{d_{h \cdot \hat{\sigma}(j)}}=\frac{S_{i, \hat{\sigma}(j)} \psi(h)}{d_{\hat{\sigma}(j)}}=\sigma\left(\frac{S_{i, j} \psi(h)}{d_{j}}\right)=\sigma\left(\frac{S_{i, h \cdot j}}{d_{h \cdot j}}\right)=\frac{S_{i, \hat{\sigma}(h \cdot j)}}{d_{\hat{\sigma}(h \cdot j)}} .
$$

Since $\left\{\phi_{j}(i):=\frac{S_{i, j}}{d_{j}} \mid j \in \operatorname{Irr}(\mathcal{C})\right\}$ is a linearly independent set of characters of $K_{0}(\mathcal{C})$, this implies $h \cdot \hat{\sigma}(j)=\hat{\sigma}(h \cdot j)$ for all $j \in \operatorname{Irr}(\mathcal{C})$, i.e. the actions of $H$ and $\operatorname{Gal}(\mathcal{C})$ on $\operatorname{Irr}(\mathcal{C})$ commute.

4.2 Support cycles Let $n$ be a positive integer, and $p$ an odd prime number. Then, there are unique non-negative integers $a, q$ with $p \nmid q$ such that $n=p^{a} q$. For the remainder discussion of this paper, we will abbreviate $v_{p}(n)=a$ and $k_{p}(n)=\frac{\varphi\left(p^{a}\right)}{2}$, where $\varphi$ is the Euler's totient function. For any element $x$ in a group of finite order, we will denote $\operatorname{ord}_{p}(x)=p^{v_{p}(\operatorname{ord}(x))}$, the maximal $p$-power factor of $\operatorname{ord}(x)$.

Let $N=\mathrm{FSexp}(\mathcal{C})$ of a modular category $\mathcal{C}$. By $[12]$, the level $n=\operatorname{ord}(t)$ of any normalized modular data $(s, t)$ satisfies $N|n| 12 N$. In particular, the modular data $(s, t)$ is defined over the cyclotomic field $\mathbb{Q}_{12 N}$.

Suppose $p$ is an odd prime and $12 N=p^{b} m$ for some positive integer $b$ and $m$ with $p \nmid m$. There exists $\sigma \in \operatorname{Gal}\left(\mathbb{Q}_{a b}\right)$, where $\mathbb{Q}_{a b}$ is the abelian closure of $\mathbb{Q}$ in $\mathbb{C}$, such that $\sigma$ fixes $\mathbb{Q}_{m}$ and $\left.\sigma\right|_{\mathbb{Q}_{p^{b}}}$ is a generator of the cyclic group $\operatorname{Gal}\left(\mathbb{Q}_{p^{b}} / \mathbb{Q}\right) \cong \mathbb{Z}_{p^{a}}^{*}$. We call such $\sigma$ p-automorphisms of $\mathcal{C}$.

Associated with a $p$-automorphism $\sigma$ is a permutation $\hat{\sigma}$ on $\operatorname{Irr}(\mathcal{C})$, which can be decomposed into a product of unique disjoint cycles. For notational convenience we identify a cycle with its support. Suppose $C$ is a cycle in this decomposition and $j \in C$ and $\operatorname{ord}_{p}\left(t_{j}\right)=\ell$, where $t_{j}$ is the $j$-th diagonal entry of $t$ in a normalized modular data $(s, t)$. Then, by Galois symmetry (cf. [12]),

$$
\ell=\operatorname{ord}_{p}\left(\sigma^{2}\left(t_{j}\right)\right)=\operatorname{ord}_{p}\left(t_{\hat{\sigma}(j)}\right) \text {. }
$$


Thus, $\operatorname{ord}_{p}\left(t_{i}\right)=\ell$ for all $i \in C$, and we call $C$ a $p^{\ell}$-support cycle of $\sigma$ if $\ell>0$. If $a=v_{p}(n)>0$, a $p^{a}$-support cycle of $\sigma$ is called a maximal support cycle of $\sigma$.

Remark 4.5. If $\left(s^{\prime}, t^{\prime}\right)$ is another normalized modular data of $\mathcal{C}$, then $\left(s^{\prime}, t^{\prime}\right)=\left(s x^{-3}, t x\right)$ for some 12-th root of unity $x$. Therefore, all the $p^{\ell}$-support cycles are independent of the choice of the normalized pair $(s, t)$ if $p>3$ or $\ell>1$. However, the 1 and 3support cycles of a 3-automorphism could be changed if $3 \mid \operatorname{ord}(x)$. We can resolve this particular ambiguity by using only a fixed family of normalizations for some modular categories.

Lemma 4.6. Suppose $\mathcal{C}$ is a modular category and $(s, t)$ a normalized modular data of level $n=p^{a} q$ for some odd prime $p$ and positive integers $a, q$ such that $p \nmid q$. Then $\sigma$ admits a maximal support cycle. For any $p^{\ell}$-support cycle $C$ of $\sigma$ with $1 \leq \ell \leq a$, we have

$$
\frac{\varphi\left(p^{\ell}\right)}{2}|\operatorname{ord}(C)| 2 k_{p}(n)=\varphi\left(p^{a}\right) .
$$

In particular, for the maximal support cycle we have $k_{p}(n)|\operatorname{ord}(\hat{\sigma})| 2 k_{p}(n)$.

If 1 is in a $p^{\ell}$-support cycle $C$ of $\sigma$ and $t_{1}=x \zeta$ for some $x, \zeta \in \mathbb{C}$ with $\operatorname{ord}(\zeta)=p^{\ell}$ and $x^{q}=1$, then $t_{\hat{\sigma}^{j}(1)}=x \sigma^{2 j}(\zeta)$ for all $j$. In particular, $\operatorname{ord}_{p}\left(t_{j}\right)=p^{\ell}$ for all $j \in C$.

Proof. Let $k=k_{p}(n)$. Since $\operatorname{ord}\left(\left.\sigma\right|_{\mathbb{Q}_{p^{a}}}\right)=2 k, \operatorname{ord}(\hat{\sigma}) \mid 2 k$ and the length of each disjoint cycle of $\hat{\sigma}$ is a divisor of $2 k$.

Since $\operatorname{ord}_{p}(n)=a$, there exists $j_{0} \in \operatorname{Irr}(\mathcal{C})$ such that $\operatorname{ord}_{p}\left(t_{j_{0}}\right)=p^{a}$. Therefore, the disjoint cycle of $\hat{\sigma}$ containing $j_{0}$ is a $p^{a}$-support cycle of $\sigma$.

Suppose $C$ is a $p^{\ell}$-support cycle of $\sigma$, for some $\ell \leq a$, with $1 \in C$. Then $t_{1}=x \zeta$ for some $x, \zeta \in \mathbb{C}$ such that $\operatorname{ord}(\zeta)=p^{\ell}$ and $x^{q}=1$. Let $k^{\prime}=\varphi\left(p^{\ell}\right) / 2$. Note that

$$
t_{1}, \sigma^{2}\left(t_{1}\right), \ldots, \sigma^{2\left(k^{\prime}-1\right)}\left(t_{1}\right)
$$

are distinct, and $\sigma^{2 k^{\prime}}\left(t_{1}\right)=t_{1}$. By Galois symmetry,

$$
\sigma^{2 j}\left(t_{1}\right)=t_{\hat{\sigma}^{j}(1)}=x \sigma^{2 j}(\zeta) \text { for all } j \text {. }
$$

In particular, $1, \hat{\sigma}(1), \ldots, \hat{\sigma}^{k^{\prime}-1}(1)$ are distinct, and $\operatorname{ord}_{p}\left(t_{\hat{\sigma}^{j}(1)}\right)=p^{\ell}$. Therefore, the length of the cycle $C$ is a multiple $k^{\prime}$ and so

$$
k^{\prime}|\operatorname{ord}(C)| \operatorname{ord}(\hat{\sigma}) \mid 2 k .
$$

For $\ell=a$, we find $k|\operatorname{ord}(C)| \operatorname{ord}(\hat{\sigma}) \mid 2 k$.

If the anomaly $\alpha=p_{+} / p_{-}$of $\mathcal{C}$ is such that $3 \nmid \operatorname{ord}(\alpha)$, one can choose a 6 -th root $\lambda$ of $\alpha$ such that $\frac{p_{+}}{\lambda^{3}}=\sqrt{\operatorname{dim\mathcal {C}}}$ and $3 \nmid \operatorname{ord}(\lambda)$. The 3 -support cycle of a 3-automorphism of 
$\mathcal{C}$ is independent of the choice of any of these normalizations. Moreover, the level $n$ of any of these normalized modular satisfies $\operatorname{FSexp}(\mathcal{C})|n| 4 \operatorname{FSexp}(\mathcal{C})$.

4.3 Support cycles for weakly integral modular categories We are particularly interested in weakly integral modular categories as their anomaly $\alpha=\frac{p_{+}}{p_{-}}$can only be an 8-th root of unity (cf. [12]). Let $D=\sqrt{\operatorname{dim\mathcal {C}}}$ be the positive square root. For any weakly integral modular category $\mathcal{C}$, we only consider the normalized modular data $(s, t)=\left(\frac{S}{D}, \frac{1}{\lambda} T\right)$ with $\lambda=\sqrt{\alpha}^{5}$ where $\sqrt{\alpha} D=p_{+}$. A 3-support cycle for a 3automorphism of $\mathcal{C}$ for this normalized modular data is then well-defined and we have a more refined statement for $p^{\ell}$-support cycles for a weakly integral modular category.

Lemma 4.7. Suppose $p$ is an odd prime and $\sigma$ is a p-automorphism of a weakly integral modular category $\mathcal{C}$ such that $v_{p}(\operatorname{FS} \exp (\mathcal{C}))=a>0$. Then, the following statements are equivalent for any positive integer $\ell \leq a$ :

(i) $C$ is a $p^{\ell}$-support cycle of $\sigma$;

(ii) $\operatorname{ord}_{p}\left(\theta_{j}\right)=p^{\ell}$ for some $j \in C$;

(iii) $\operatorname{ord}_{p}\left(\theta_{j}\right)=p^{\ell}$ for all $j \in C$.

If $C=(12 \ldots l)$ and $\theta_{1}=x \zeta$ for some $x, \zeta \in \mathbb{C}$ with $\operatorname{ord}(\zeta)=p^{\ell}$ and $x^{q}=1$ with $p \nmid q$, then $\theta_{j}=x \sigma^{2(j-1)}(\zeta)$ for $j=1, \ldots, l$. In particular, if $C_{0}$ is the cycle of $\sigma$ containing 0 , then $\theta_{j}=1$ for all $j \in C_{0}$ and $\operatorname{ord}\left(C_{0}\right) \leq 2$. Moreover, $X_{\hat{\sigma}(0)}$ is a self-dual invertible object.

Proof. Let $t=\sqrt{\alpha}^{5} T$ where $\sqrt{\alpha}=p_{+} / D$ is a 16-th root of unity. Then $\operatorname{ord}_{p}\left(t_{j}\right)=$ $\operatorname{ord}_{p}\left(\theta_{j}\right)$ for all $j$, and so the equivalence of the three conditions follows from Lemma 4.6. Note that $t_{0}$ is a 16 -th root but $p \mid \operatorname{ord}\left(t_{j}\right)$ for any $j$ in a $p^{\ell}$-support cycle $C$ of $\sigma(\ell>0)$. Therefore, $0 \notin C$. If $C=(12 \ldots l)$ and $\theta_{1}=x \zeta$ for some $x, \zeta \in \mathbb{C}$ with $\operatorname{ord}(\zeta)=p^{\ell}$ and $x^{q}=1$, then $t_{1}=\sqrt{\alpha}^{5} x \zeta$ and so $t_{j}=\sqrt{\alpha}^{5} x \sigma^{2(j-1)}(\zeta)$. Thus, $\theta_{j}=x \sigma^{2(j-1)}(\zeta)$.

Since $\mathcal{C}$ is weakly integral, $\sigma^{2}\left(d_{i}\right)=d_{i}$ for all $i \in \operatorname{Irr}(\mathcal{C})$. This implies the length of $C_{0}$ is at most 2. Since $\theta_{0}=1, \theta_{\hat{\sigma}(0)}=1$. If $g=\hat{\sigma}(0) \neq 0$, then $S_{g, j}= \pm S_{0, j}= \pm d_{j}$ for all $j \in \operatorname{Irr}(\mathcal{C})$. By $\left[19\right.$, Lem. 6.1], $X_{g}$ is invertible. Since $g$-th row of the $S$-matrix is real, $X_{g}$ is self-dual.

Theorem 4.8. Let $\mathcal{C}$ be a weakly integral modular category with $N=\operatorname{FSexp}(\mathcal{C})$ which has a simple odd prime factor $p$. Suppose a p-automorphism $\sigma$ of $\mathcal{C}$ has only one $p$ support cycle $C_{1}=(1 \ldots l)$. Then: 
(i) $\operatorname{dim} \mathcal{C}=\left(\frac{l}{p-1}\right)^{2} d_{1}^{4} p, \sigma\left(d_{1}\right)=d_{1}, \sigma\left(p_{+}\right)=-p_{+}$and $\theta_{1}=x \zeta$, for some $x, \zeta \in \mathbb{C}$ with $\operatorname{ord}(\zeta)=p$ and $x^{16}=1$. In particular, if $l=\frac{p-1}{2}$, then $2 \mid d_{1}^{2}$.

(ii) If $\mathcal{C}$ is integral and $\operatorname{Gal}(\mathcal{C})$ is generated by $\hat{\sigma}$, then $\hat{\sigma}$ is a cycle of length $p-1$ and $\mathcal{C}$ is pointed of rank $p$. In particular, $\mathcal{C}$ is a cyclic modular category.

(iii) If $\mathcal{C}$ is a strictly weakly integral and $\operatorname{Gal}(\mathcal{C})$ is generated by $\hat{\sigma}$, then $\hat{\sigma}=$ $(0,1)\left(2, \ldots, \frac{p+1}{2}\right)$, up to a relabeling of the simple objects, and $\mathcal{C}$ is a prime modular category of $\operatorname{dim} \mathcal{C}=4 p$. In particular, $\mathcal{C}$ is of rank $\frac{p+7}{2}$, and equivalent to a $\mathbb{Z}_{2}$-equivariantization of a Tambara-Yamagami category associated to the group $\mathbb{Z}_{p}$.

Proof. Since $\mathcal{C}$ is pseudo-unitary, we may assume $d_{i}>0$ for all $i \in \operatorname{Irr}(\mathcal{C})$ by using the canonical pivotal structure of $\mathcal{C}$. Suppose $N=p q$ for some non-negative integer $q$ relatively prime to $p$. Then $\theta_{1}=x \zeta$ for some $x, \zeta \in \mathbb{C}$ with $\operatorname{ord}(\zeta)=p$ and $x^{q}=1$. By Lemma 4.7, $\theta_{j}=x \sigma^{2(j-1)}(\zeta)$, for $j=1, \ldots, l$, and $l=p-1$ or $\frac{p-1}{2}$. Suppose $\hat{\sigma}=C_{0} C_{1} C_{2} \cdots C_{m}$ where $C_{0}$ is the cycle containing 0 . Note that $d_{j}=d_{C_{i}}$ for all $j \in C_{i}$. In particular, $d_{C_{1}}=d_{1}$. Since $C_{1}$ is the only $p$-support cycle of $\sigma$, we also have $\theta_{j}=\theta_{C_{i}} \in \mathbb{Z}\left[\zeta_{q}\right]$ for all $j \in C_{i}$ if $i \neq 1$. Let us denote $l_{i}=\operatorname{ord}\left(C_{i}\right)$ and $C_{i}=$ $\left(c_{i} \hat{\sigma}\left(c_{i}\right) \hat{\sigma}^{2}\left(c_{i}\right) \ldots\right)$. Now we consider the twist equation (2.1) for $S_{0 j}$ :

$$
\begin{aligned}
p_{+} d_{j} \bar{\theta}_{j} & =d_{1} \sum_{r=1}^{l} S_{j r} \theta_{r}+\sum_{i \neq 1} d_{C_{i}} \theta_{C_{i}} \sum_{r \in C_{i}} S_{j r} \\
& =d_{1}^{2} x \sum_{r=0}^{l-1} \frac{S_{j, 1+r}}{d_{1}} \sigma^{2 r}(\zeta)+\sum_{i \neq 1} d_{C_{i}}^{2} \theta_{C_{i}} \sum_{r \in C_{i}} \frac{S_{j r}}{d_{r}} .
\end{aligned}
$$

For any $i$, we denote

$$
S_{j, C_{i}}=\sum_{r \in C_{i}} \frac{S_{j r}}{d_{r}}=\sum_{r=0}^{l_{i}-1} \sigma^{r-1}\left(\frac{S_{j, c_{i}}}{d_{C_{i}}}\right) .
$$

Then $\sigma$ fixes $S_{j, C_{i}}$, and so $\sum_{i \neq 1} d_{C_{i}}^{2} \theta_{C_{i}} S_{j, C_{i}}$, and 4.3 becomes

$$
p_{+} d_{j} \bar{\theta}_{j}=d_{1}^{2} x \sum_{r=0}^{l-1} \sigma^{r}\left(\frac{S_{j, 1}}{d_{1}}\right) \sigma^{2 r}(\zeta)+\sum_{i \neq 1} d_{C_{i}}^{2} \theta_{C_{i}} S_{j, C_{i}} .
$$


Therefore, for $j=0$, we have

$$
\begin{aligned}
\sqrt{\alpha} D=p_{+} & =d_{1}^{2} x \sum_{r=0}^{l-1} \sigma^{2 r}(\zeta)+\sum_{i \neq 1} d_{C_{i}}^{2} \theta_{C_{i}} l_{i} \\
& =\frac{2 l d_{1}^{2} x}{p-1}\left(\frac{-1 \pm \varepsilon \sqrt{p}}{2}\right)+\sum_{i \neq 1} d_{C_{i}}^{2} \theta_{C_{i}} l_{i} \\
& = \pm \frac{l d_{1}^{2} x}{p-1} \varepsilon \sqrt{p}+\frac{-l d_{1}^{2} x}{p-1}+\sum_{i \neq 1} d_{C_{i}}^{2} \theta_{C_{i}} l_{i}
\end{aligned}
$$

where $\varepsilon=\sqrt{\left(\frac{-1}{p}\right)}$ and $(\dot{\bar{p}})$ is the Legendre symbol. Since $\alpha=p_{+} / p_{-} \in \mathbb{Q}_{N}$ and $\alpha^{8}=1, \alpha \in \mathbb{Q}_{q}$ and so $p_{+}^{2} \in \mathbb{Q}_{q}$. Note that $\mathbb{Q}(\varepsilon \sqrt{p})$ is the unique quadratic subfield of $\mathbb{Q}_{p}$. Therefore, $p_{+} \in \mathbb{Q}_{q}$ or $p_{+} \in \varepsilon \sqrt{p} \cdot \mathbb{Q}_{q}^{\times}$. Since $\frac{l d_{1}^{2} x}{p-1} \neq 0$ and $\{1, \varepsilon \sqrt{p}\}$ is linearly independent over $\mathbb{Q}_{q}$, it follows from 4.7 that

$$
\sqrt{\alpha} D= \pm \frac{d_{1}^{2} x l}{p-1} \varepsilon \sqrt{p} \text { and } \frac{l d_{1}^{2} x}{p-1}=\sum_{i \neq 1} d_{C_{i}}^{2} \theta_{C_{i}} l_{i} .
$$

This implies $\alpha=x^{2}\left(\frac{-1}{p}\right), \operatorname{dim} \mathcal{C}=\left(\frac{l}{p-1}\right)^{2} d_{1}^{4} p$, and $\sigma\left(p_{+}\right)=-p_{+}$. Therefore,

$$
2 p_{+}=d_{1}^{2} x \sum_{r=0}^{l-1}\left(\sigma^{2 r}(\zeta)-\sigma^{2 r+1}(\zeta)\right) .
$$

We now show that $d_{1}$ is fixed by $\sigma$. Suppose $\sigma\left(d_{1}\right) \neq d_{1}$, then $\sigma\left(d_{1}\right)=-d_{1}$ as $d_{1}$ is square root of an integer. Since $\sigma^{2}\left(d_{j}\right)=d_{j}$ for all $j \in \operatorname{Irr}(\mathcal{C})$, we get $l_{0}=2$ and we write $C_{0}=(0, \hat{\sigma}(0))$. Thus,

$$
S_{1, C_{0}}=d_{1}+S_{1, \hat{\sigma}(0)}=d_{1}+\left(-d_{1}\right)=0 .
$$

By Lemma 4.7, $\theta_{\hat{\sigma}(0)}=1$. Summing (4.4) for $j=0, \hat{\sigma}(0)$, we find

$$
2 p_{+}=\sum_{i \neq 1} d_{C_{i}}^{2} \theta_{C_{i}}\left(S_{0, C_{i}}+S_{\hat{\sigma}(0), C_{i}}\right) .
$$

However, this implies $p_{+}$is fixed by $\sigma$, which is a contradiction. Therefore, $\sigma$ fixes $d_{1}$. This completes the proof of (i).

We now assume $\operatorname{Gal}(\mathcal{C})=\langle\hat{\sigma}\rangle$ for the remainder proofs of (ii) and (iii). Then, it follows from (i) that $d_{1}, S_{j, C_{i}} \in \mathbb{Z}$ for all $i$, and $d_{C_{i}}^{2} \theta_{C_{i}} S_{j, C_{i}} \in \mathbb{Z}\left[\zeta_{q}\right]$ for $i \neq 1$.

Let $I=\left\{j \in \operatorname{Irr}(\mathcal{C}) \mid d_{i} \in \mathbb{Z}\right\}, I^{c}=\operatorname{Irr}(\mathcal{C}) \backslash I$ and $\tilde{I}=I \backslash\{1, \ldots, l\}$, and let $\langle I\rangle$ be the tensor subcategory generated by $I$. For any disjoint cycle $C_{a}$ of $\sigma$, we write $C_{a} \subset \tilde{I}$ if 
all the entries of $C_{a}$ are in $\tilde{I}$. Similarly, we write $C_{a} \subset I^{c}$ if all the entries of $C_{a}$ are in $I^{c}$.

Suppose $C_{a} \subset \tilde{I}$. By summing $j \in C_{a}$ of 4.4 , we find

$$
l_{a} p_{+} d_{C_{a}} \bar{\theta}_{C_{a}}=d_{1} d_{C_{a}} x S_{1, C_{a}} \sum_{r=0}^{l-1} \sigma^{2 r}(\zeta)+\sum_{\substack{i \neq 1 \\ j \in C_{a}}} d_{C_{i}}^{2} \theta_{C_{i}} S_{j, C_{i}}
$$

Since the last term of this equation is fixed by $\sigma$, the difference of this equation and its image under $\sigma$ is given by

$$
2 l_{a} p_{+} d_{C_{a}} \bar{\theta}_{C_{a}}=d_{1} d_{C_{a}} x S_{1, C_{a}} \sum_{r=0}^{l-1} \sigma^{2 r}(\zeta)-\sigma^{2 r+1}(\zeta)
$$

By (4.9), we find $S_{1, C_{a}}=l_{a} d_{1} \bar{\theta}_{C_{a}}$. Hence $\theta_{C_{a}}= \pm 1$ and $S_{r, C_{a}}=l_{a} d_{1} \bar{\theta}_{C_{a}}$, for all $r \in C_{1}$. Now $\left|S_{r, j}\right| / d_{C_{a}} \leq d_{1}$ for $j \in C_{a}$ and $r \in C_{1}$ as $S_{r, j} / d_{C_{a}}$ and $d_{1}$ are eigenvalues of the same matrix with $d_{1}$ the Frobenius-Perron eigenvalue. Thus we find

$$
S_{r, j}=d_{r} d_{j} \theta_{C_{a}} \in \mathbb{Z}
$$

for $j \in C_{a}$ and $r \in C_{1}$.

Suppose $C_{b} \subset \tilde{I}$. For $j \in C_{b}$, we have $S_{j, C_{a}}=S_{c_{b}, C_{a}}$. For any $j^{\prime} \in C_{a}$, we have the twist equation:

$$
p_{+} S_{j, j^{\prime}}=\theta_{j} \theta_{j^{\prime}}\left(x \sum_{r=0}^{l-1} S_{j, 1+r} S_{j^{\prime}, 1+r} \sigma^{2 r}(\zeta)+\sum_{i \neq 1} \sum_{r \in C_{i}} S_{j r} S_{j^{\prime} r} \theta_{C_{i}}\right) .
$$

Therefore,

$$
\begin{aligned}
& \sum_{j \in C_{b}} \sum_{j^{\prime} \in C_{a}} p_{+} S_{j, j^{\prime}}=d_{C_{a}} l_{b} p_{+} S_{c_{b}, C_{a}} \\
= & \theta_{C_{b}} \theta_{C_{a}}\left(x d_{C_{a}} d_{C_{b}} \sum_{r=0}^{l-1} S_{1+r, C_{b}} S_{1+r, C_{a}} \sigma^{2 r}(\zeta)+d_{C_{a}} d_{C_{b}} \sum_{i \neq 1} \sum_{r \in C_{i}} S_{r, C_{b}} S_{r, C_{a}} \theta_{C_{i}}\right) \\
= & \theta_{C_{b}} \theta_{C_{a}}\left(x d_{C_{a}} d_{C_{b}} S_{1, C_{b}} S_{1, C_{a}} \sum_{r=0}^{l-1} \sigma^{2 r}(\zeta)+d_{C_{a}} d_{C_{b}} \sum_{i \neq 1} \sum_{r \in C_{i}} S_{r, C_{b}} S_{r, C_{a}} \theta_{C_{i}}\right) \\
= & x d_{C_{a}} d_{C_{b}} l_{a} l_{b} d_{1}^{2} \sum_{r=0}^{l-1} \sigma^{2 r}(\zeta)+\theta_{C_{b}} \theta_{C_{a}} d_{C_{a}} d_{C_{b}} \sum_{i \neq 1} \sum_{r \in C_{i}} S_{r, C_{b}} S_{r, C_{a}} \theta_{C_{i}} .
\end{aligned}
$$


Since $S_{c_{b}, C_{a}}$ and $\theta_{C_{b}} \theta_{C_{a}} d_{C_{a}} d_{C_{b}} \sum_{i \neq 1} \sum_{r \in C_{i}} S_{r, C_{b}} S_{r, C_{a}} \theta_{C_{i}}$ are fixed by $\sigma$, we find

$$
2 d_{C_{a}} l_{b} p_{+} S_{c_{b}, C_{a}}=x d_{C_{a}} d_{C_{b}} l_{a} l_{b} d_{1}^{2} \sum_{j=0}^{l-1}\left(\sigma^{2 j}(\zeta)-\sigma^{2 j+1}(\zeta)\right)=2 p_{+} d_{C_{a}} d_{C_{b}} l_{a} l_{b} .
$$

Hence, $S_{c_{b}, C_{a}}=l_{a} d_{C_{b}}$. By the same argument as above, $S_{j, j^{\prime}}=d_{C_{a}} d_{C_{b}}=d_{j} d_{j^{\prime}}$ for $j \in C_{b}$ and $j^{\prime} \in C_{a}$. For any $j, j^{\prime} \in \tilde{I}$, there exist $C_{a}, C_{b} \subset \tilde{I}$ such that $j^{\prime} \in C_{a}$ and $j \in C_{b}$. Hence we have

$$
S_{j, j^{\prime}}=d_{j} d_{j^{\prime}}, \text { for all } j, j^{\prime} \in \tilde{I} .
$$

By summing (4.4) over all $j \in C_{b}$, we obtain

$$
\begin{aligned}
l_{b} p_{+} d_{C_{b}} \theta_{C_{b}} & =d_{1} d_{C_{b}} x \sum_{r=0}^{l-1} S_{r, C_{b}} \sigma^{2 r}(\zeta)+\sum_{j \in C_{b}} \sum_{i \neq 1} d_{C_{i}}^{2} \theta_{C_{i}} S_{j, C_{i}} \\
& =d_{1} d_{C_{b}} x S_{1, C_{b}} \sum_{r=0}^{l-1} \sigma^{2 r}(\zeta)+l_{b} d_{C_{b}} \sum_{C_{i} \subset \tilde{I}} d_{C_{i}}^{2} \theta_{C_{i}} l_{i}+\sum_{j \in C_{b}} \sum_{C_{i} \subset I^{c}} d_{C_{i}}^{2} \theta_{C_{i}} S_{j, C_{i}} \\
& =l_{b} d_{1}^{2} d_{C_{b}} \theta_{C_{b}} x \sum_{r=0}^{l-1} \sigma^{2 r}(\zeta)+l_{b} d_{C_{b}} \sum_{C_{i} \subset \tilde{I}} d_{C_{i}}^{2} \theta_{C_{i}} l_{i}+\sum_{j \in C_{b}} \sum_{C_{i} \subset I^{c}} d_{C_{i}}^{2} \theta_{C_{i}} S_{j, C_{i}}
\end{aligned}
$$

or

$$
p_{+}=d_{1}^{2} x \sum_{r=0}^{l-1} \sigma^{2 r}(\zeta)+\theta_{C_{b}} \sum_{C_{i} \subset \tilde{I}} d_{C_{i}}^{2} \theta_{C_{i}} l_{i}+\frac{\theta_{C_{b}}}{l_{b} d_{C_{b}}} \sum_{\substack{C_{i} \subset I^{c} \\ j \in C_{b}}} d_{C_{i}}^{2} \theta_{C_{i}} S_{j, C_{i}}
$$

(ii) If $\mathcal{C}$ is integral, then $I=\operatorname{Irr}(\mathcal{C})$ and so

$$
p_{+}=d_{1}^{2} x \sum_{r=0}^{l-1} \sigma^{2 r}(\zeta)+\theta_{C_{b}} \sum_{i \neq 1} d_{C_{i}}^{2} \theta_{C_{i}} l_{i} .
$$

Since $\sum_{i \neq 1} d_{C_{i}}^{2} \theta_{C_{i}} l_{i} \neq 0$ (cf. 4.8) ), by comparing this equation with 4.5), we have $\theta_{C_{b}}=1$ for $C_{b} \in \tilde{I}$. It follows from 4.10 that $S_{r, j}=d_{r} d_{j}$ for $r \in C_{1}$. Therefore, if $j \notin C_{1}, S_{i, j}=d_{i} d_{j}$ for all $i \in \operatorname{Irr}(\mathcal{C})$. This forces $j=0$ and so $\operatorname{Irr}(\mathcal{C})=\{0, \ldots l\}$. By (i) we have

$$
\left(\frac{l}{p-1}\right)^{2} d_{1}^{4} p=1+l d_{1}^{2} .
$$

The equation has no integral solution for $d_{1}$ if $l=\frac{p-1}{2}$. For $l=p-1$, the only integer solution is $d_{1}=1$. Thus, $\mathcal{C}$ is pointed of rank $p$, and the proof of (ii) is completed. 
(iii) Now, we assume $\mathcal{C}$ is strictly weakly integral. Then $I^{c} \neq \emptyset$ and so $C_{0}$ must have length $>1$. By Lemma 4.7, $C_{0}=(0, g), \theta_{g}=1$ and $g \in G(\mathcal{C})$ is of order 2, where $g=\hat{\sigma}(0)$. Let $H=\{0, g\}$ and $\mathcal{C}(H)$ the fusion category generated by $X_{0}$ and $X_{g}$. Then $\operatorname{dim} \mathcal{C}(H)=2$ and $\mathcal{C}(H)$ is a subcategory of the centralizer $\langle I\rangle^{\prime}$ by (4.10) and 4.11, where $\langle I\rangle$ is the subcategory generated by $X_{i}, i \in I$. We proceed to show that the centralizer $\langle I\rangle^{\prime}=\mathcal{C}(H)$ by showing that $\operatorname{dim}\langle I\rangle^{\prime}=2$.

Since $\operatorname{Gal}(\mathcal{C})$ is cyclic, $\mathbb{K}_{\mathcal{C}}$ has exactly one quadratic extension over $\mathbb{Q}$. Therefore, there exists a square-free positive $M$ such that $d_{j}=\alpha_{j} \sqrt{M}$, for all $j \in I^{c}$ and positive integer $\alpha_{j}$. Let $\mathcal{C}_{0}=\langle I\rangle$ and let $\mathcal{C}_{1}$ be the subcategory of $\mathcal{C}$ generated by $X_{j}$ for $j \in I^{c}$. Then, $\mathcal{C}=\mathcal{C}_{0} \oplus \mathcal{C}_{1}$ defines a faithful $\mathbb{Z}_{2}$-grading on $\mathcal{C}$. Thus, $\operatorname{dim}\langle I\rangle=\operatorname{dim} \mathcal{C} / 2$. By $[23]$, $\operatorname{dim}\langle I\rangle^{\prime}=2$.

Next, we would like to prove that $\tilde{I}=H$. Now we consider the twist equation (2.1) for $S_{g, j}$ with $j \in \tilde{I}$ :

$$
p_{+} d_{j} \bar{\theta}_{j}=d_{1} \sum_{r=1}^{l} S_{j, r} \theta_{r}+\sum_{C_{i} \subset \tilde{I}} d_{C_{i}} \theta_{C_{i}} \sum_{r \in C_{i}} S_{j, r}-\sum_{C_{i} \subset I^{c}} d_{C_{i}} \theta_{C_{i}} \sum_{r \in C_{i}} S_{j, r} .
$$

By comparing this equation with 4.2 , we find

$$
\sum_{C_{i} \subset I^{c}} d_{C_{i}}^{2} \theta_{C_{i}} S_{j, C_{i}}=\sum_{C_{i} \subset I^{c}} d_{C_{i}} \theta_{C_{i}} \sum_{r \in C_{i}} S_{j, r}=0 \text { for all } j \in \tilde{I} \text {. }
$$

By setting $j=0$ in 4.4 , we obtain $\sum_{C_{i} \subset I^{c}} d_{C_{i}}^{2} \theta_{C_{i}} l_{i}=0$. Therefore, by comparing 4.5 and (4.12), we have $\theta_{j}=1$, for all $j \in \tilde{I}$. This implies $S_{i j}=d_{i} d_{j}$, for all $i \in I$ and $j \in \tilde{I}$. Hence $X_{j} \in C_{\mathcal{C}}(\langle I\rangle)$, for $j \in \tilde{I}$. Therefore, $\tilde{I}=H$ and so $I=H \cup\{1, \ldots, l\}$.

By (i), we now have an equation on the dimension of $\langle I\rangle$ :

$$
\left(\frac{l}{p-1}\right)^{2} \frac{d_{1}^{4} p}{2}=\frac{\operatorname{dim} \mathcal{C}}{2}=2+l d_{1}^{2} .
$$

This equation has no integral solution for $d_{1}$ if $l=p-1$. This implies $l=\frac{p-1}{2}$, and so $d_{1}=2$ is the only integer solution. Thus, $\operatorname{dim} \mathcal{C}=4 p$. It follows from Theorem 3.1 (b) that $\mathcal{C}$ is a $\mathbb{Z}_{2}$-equivariantization of a Tambara-Yamagami category associated to the group $\mathbb{Z}_{p}$. This completes the proof of (iii).

Lemma 4.9. Suppose $\mathcal{C}$ is a weakly integral modular category such that $v_{p}(\operatorname{FS} \exp (\mathcal{C}))=$ 1 , for some odd prime $p$. Let $\sigma$ be a p-automorphism of $\mathcal{C}$. If $\sigma$ has exactly two $p$-support cycles and they both have length $k=\frac{p-1}{2}$, say $C_{1}=(1 \ldots k)$ and $C_{2}=(k+1 \ldots 2 k)$, then either

(i) $v_{p}(\operatorname{dim} \mathcal{C})$ is even and $d_{1}=\cdots=d_{p-1}$, or 
(ii) $v_{p}(\operatorname{dim} \mathcal{C})$ is odd and $\operatorname{dim} \mathcal{C}=\frac{1}{4}\left(d_{1}^{4}+d_{2 k}^{4}+\epsilon d_{1}^{2} d_{2 k}^{2}\right) p$, for some $\epsilon \in\{0,1,-1\}$.

Proof. Suppose $\hat{\sigma}=(1 \ldots k)(k+1 \ldots 2 k) \prod_{i} Z_{i}$, for some disjoint cycles $Z_{i}$. Let $J=$ $\operatorname{Irr}(\mathcal{C}) \backslash\{1, \ldots, 2 k\}$. Since none of the elements of $J$ are contained in any support cycles of $\sigma$, it follows that $\sigma\left(\theta_{j}\right)=\theta_{j}$, for $j \in J$. Now we assume $\operatorname{FSexp}(\mathcal{C})=p q, \theta_{1}=x \zeta$ and $\theta_{2 k}=y \zeta^{\ell}$, for some $x, y, \zeta \in \mathbb{C}$ and some positive integer $\ell<p$ such that $x^{q}=y^{q}=1$ and $\operatorname{ord}(\zeta)=p$. Define $u=\sum_{i=1}^{k-1} \sigma^{2 i}(\zeta)=\frac{-1 \pm \varepsilon \sqrt{p}}{2}, u^{\prime}=\sum_{i=1}^{k-1} \sigma^{2 i}\left(\zeta^{\ell}\right)=\frac{-1 \pm\left(\frac{\ell}{p}\right) \varepsilon \sqrt{p}}{2}$, where $\varepsilon=\sqrt{\left(\frac{-1}{p}\right)}$. Then

$$
\begin{aligned}
\sqrt{\alpha} D=p_{+} & =d_{1}^{2} x u+d_{2 k}^{2} y u^{\prime}+\sum_{j \in J} d_{j}^{2} \theta_{j} \\
& =\frac{ \pm 1}{2}\left(d_{1}^{2} x+\left(\frac{\ell}{p}\right) d_{2 k}^{2} y\right) \varepsilon \sqrt{p}-\frac{1}{2}\left(d_{1}^{2} x+d_{2 k}^{2} y\right)+\sum_{j \in J} d_{j}^{2} \theta_{j} .
\end{aligned}
$$

Note that $\sum_{j \in J} d_{j}^{2} \theta_{j} \in \mathbb{Q}_{q}$. If $v_{p}(\operatorname{dim} \mathcal{C})$ is even, then $p_{+} \in \mathbb{Q}_{q}$. The $\mathbb{Q}_{q}$-linear independence of $\{1, \sqrt{p}\}$ implies $d_{1}^{2} x+\left(\frac{\ell}{p}\right) d_{2 k}^{2} y=0$, and so $d_{1}^{2}=d_{2 k}^{2}$. On the hand, If $v_{p}(\operatorname{dim} \mathcal{C})$ is odd, then $p_{+} \notin \mathbb{Q}_{q}$, and so

$$
\sqrt{\alpha} D=\frac{ \pm 1}{2}\left(d_{1}^{2} x+\left(\frac{\ell}{p}\right) d_{p-1}^{2} y\right) \varepsilon \sqrt{p} .
$$

Hence, by considering the product of conjuagates, we have

$$
\frac{4 \operatorname{dim} \mathcal{C}}{p}=\left(d_{1}^{2}+\left(\frac{\ell}{p}\right) d_{2 k}^{2} \frac{y}{x}\right)\left(d_{1}^{2}+\left(\frac{\ell}{p}\right) d_{2 k}^{2} \frac{y}{x}\right)=d_{1}^{4}+d_{2 k}^{4}+\left(\frac{\ell}{p}\right) d_{1}^{2} d_{2 k}^{2}\left(\frac{y}{x}+\frac{x}{y}\right) .
$$

This implies $y / x$ is either a 4 -th root or a 6 -th root of unity and so we obtain

$$
\frac{4 \operatorname{dim} \mathcal{C}}{p}=d_{1}^{4}+d_{2 k}^{4}+\epsilon d_{1}^{2} d_{2 k}^{2}
$$

where $\epsilon \in\{0,1,-1\}$.

\section{WEAKLY INTEGRAL MODULAR CATEGORIES OF RANK 6 AND 7}

We now apply the results of previous sections to the classification of weakly integral modular categories of ranks 6 and 7 . 


\subsection{Weakly integral modular categories of rank 6}

Theorem 5.1. A weakly integral rank 6 modular category $\mathcal{C}$ is equivalent (as balanced braided fusion category) to one of the following:

(a) $\mathcal{I} \otimes \mathcal{P}$, with $\mathcal{I}$ an Ising modular category and $\mathcal{P}$ a cyclic modular category of $\operatorname{rank} 2$,

(b) $T Y\left(\mathbb{Z}_{5}, \chi, \nu\right)^{\mathbb{Z}_{2}}$, or

(c) a cyclic modular category of rank 6 .

Proof. If $\mathcal{C}$ is integral, it follows from $[7$, Theorem 4.2] that $\mathcal{C}$ is in fact pointed (alternative $(\mathrm{d})$ ). Therefore, we may assume that $\mathcal{C}$ is strictly weakly integral. Moreover, by Lemma 3.3, there are at least two objects with non-integral dimensions. In addition, [19, Theorem 3.10] implies that $\mathcal{C}$ is faithfully graded by an elementary 2-group, so that there are at least two invertible objects. Thus, the universal grading group $U(\mathcal{C})$ has order 2 or 4 .

If $|U(\mathcal{C})|=4$ then the two simple objects with non-integral dimension must have dimension $\sqrt{2}$, since the 4 graded components must have the same dimension and we have only 6 objects. In this case $\mathcal{C}$ is equivalent to $\mathcal{I} \otimes \mathcal{P}$, with $\mathcal{P}$ pointed of rank 2 by [24, Theorem 5.5].

If $|U(\mathcal{C})|=2$ it is enough to show that $\operatorname{dim} \mathcal{C} \in\{12,20\}$ by Theorem 3.1. Note that $\mathcal{C}_{a d}=\mathcal{C}_{e}$ is an integral premodular category containing exactly two isomorphism classes of invertible objects. Since there are at least two simple classes of objects of nonintegral dimension, the rank of $\mathcal{C}_{a d}$ is 2,3 or 4 . The first case is clearly impossible, since there are two pointed objects in $\mathcal{C}_{a d}$. If $\mathcal{C}_{a d}$ has $\operatorname{rank} 3, \operatorname{dim} \mathcal{C}=2 \operatorname{dim} \mathcal{C}_{a d}=12$ by Ostrik's classification of rank 3 premodular categories [30]. If $\operatorname{rank}\left(\mathcal{C}_{a d}\right)=4$ we have $\operatorname{dim} \mathcal{C}=2 \operatorname{dim} \mathcal{C}_{a d}=20$, by the classification of rank 4 premodular categories 44.

5.2 Weakly Integral Rank 7 The goal of this subsection is to give a classification, up to Grothendieck equivalence, of weakly integral rank 7 modular categories. Such categories obviously cannot be Deligne products of non-trivial categories. Applying the results of subsection 4.1 we have:

Proposition 5.2. Suppose $\mathcal{C}$ is a (not necessarily weakly integral) modular category of rank 7. If $\mathcal{C}$ admits a Tannakian subcategory equivalent to $\operatorname{Rep}(H)$, for some nontrivial subgroup $H$ of $G(\mathcal{C})$, then $\mathcal{C}$ is strictly weakly integral of dimension 28. 
Proof. Since $\mathcal{C}$ has a faithful $\hat{H}$-grading and $H \subset \mathcal{C}_{e}$ by Remark 4.1, the pigeonhole principle implies that $|H| \leq 4$. If $|H|=4$ then $\left|\operatorname{Irr}\left(\mathcal{C}_{\chi}\right)\right|=1$, for any non-trivial character $\chi$ of $H$, by Remark 4.1. In particular, $H$ fixes all the simple objects not in $\operatorname{Irr}\left(\mathcal{C}_{e}\right)$, but this contradicts Lemma 4.2. Thus, $|H|=2$ or 3 . Lemma 4.2 implies that if $|H|=3$ then 3|| $\operatorname{Irr}\left(\mathcal{C}_{\chi}\right) \mid$, for each non-trivial character $\chi$ of $H$. However, this means $\operatorname{rank} \mathcal{C} \geq 9$. Therefore, $|H|=2$.

Suppose $H=\{0, h\}$ and $\hat{H}=\{e, \chi\}$. In particular, $\chi(h)=-1$. In view of Lemma 4.2 . $\operatorname{Irr}\left(\mathcal{C}_{\chi}\right)$ can only have 1 or $2 H$-orbits.

We first show that $\operatorname{Irr}\left(\mathcal{C}_{\chi}\right)$ must have only one $H$-orbit. Assume the contrary. Then we have $\operatorname{Irr}\left(\mathcal{C}_{e}\right)=\left\{\mathbf{1}, h, X_{1}\right\}$ and $\operatorname{Irr}\left(\mathcal{C}_{\chi}\right)=\left\{X_{2}, X_{3}, X_{4}, X_{5}\right\}$ with $h \cdot X_{2}=X_{3}$ and $h \cdot X_{4}=X_{5}$. Note that $X_{1}$ cannot be invertible and so $X_{1}$ is fixed by $H$. In particular, $d_{1}^{2}-n d_{1}-2=0$, and $d_{1} \neq \pm 1$. Moreover, $X_{1}$ is self-dual.

Since $\mathcal{C}_{e}$ is a non-pointed premodular category with exactly two invertible classes of simple objects, [30] implies that $\operatorname{dim}\left(X_{1}\right) \in\{\sqrt{2}, 2\}$. By [14, Cor. B.12] if $\operatorname{dim}\left(X_{1}\right)=$ $\sqrt{2}$ then $\mathcal{C}_{e}$ is modular, which implies $\mathcal{C}$ is a Deligne product of two modular categories by [23. Theorem 4.2], a contradiction. If $\operatorname{dim}\left(X_{1}\right)=2$ then $\operatorname{dim} \mathcal{C}=12$, so the conditions of Theorem 3.1 are satisfied. Applying [23. Theorem 4.2] again, we see that the only alternative is that $\mathcal{C}$ is a metaplectic category of dimension 12. But such categories have rank 5 , a contradiction.

Therefore, $\operatorname{Irr}\left(\mathcal{C}_{\chi}\right)$ consists of a single $H$-orbit so that $\operatorname{Irr}\left(\mathcal{C}_{e}\right)=\left\{1, h, X_{1}, X_{2}, X_{3}\right\}$ and $\operatorname{Irr}\left(\mathcal{C}_{\chi}\right)=\left\{X_{4}, X_{5}\right\}$ with $h \cdot X_{4}=X_{5}$. Moreover, by Lemma 4.3, $\mathcal{C}_{e}$ is integral so that $\mathcal{C}$ is weakly integral. Then one of $X_{1}, X_{2}, X_{3}$ must be fixed by $H$. We may assume $X_{2}$ is fixed by $H$. Since the stabilizer of $\operatorname{Irr}\left(\mathcal{C}_{\chi}\right)$ is trivial, it follows from Lemma 4.3 that $d_{2}=2 n_{2}$, for some positive integer $n_{2}$. Moreover, $d_{1}, d_{3} \in \mathbb{Z}_{+}$. From the dimension equation we find $\operatorname{dim} \mathcal{C}=4 d_{4}^{2}, n_{2}^{2} \mid d_{4}^{2}$ and

$$
2 d_{4}^{2}=2+d_{1}^{2}+4 n_{2}^{2}+d_{3}^{2}
$$

The equation modulo 2 implies $\left(d_{1}, d_{3}, d_{4}^{2}\right) \equiv(1,1,0)$ or $(0,0,1) \bmod 2$.

We first show that $\left(d_{1}, d_{3}, d_{4}^{2}\right) \equiv(1,1,0) \bmod 2$ is not possible. Assume the contrary. Then $d_{1}, d_{3}$ are odd and so $d_{1}^{2} \mid d_{4}^{2}$. By Lemma 4.3, $X_{1}$ is not fixed by $H\left(|H|=2 \nmid d_{1}\right)$. Therefore, $h \cdot X_{1}=X_{3}$ and hence $d_{1}=d_{3}$. Now, (5.1) becomes

$$
d_{4}^{2}=1+d_{1}^{2}+2 n_{2}^{2}
$$

and hence $d_{4}^{2}$ is even. Moreover, $n_{2}^{2}$ and $d_{1}^{2}$ are relatively prime, and $d_{1}^{2} n_{2}^{2}=\operatorname{lcm}\left(d_{1}^{2}, n_{2}^{2}\right)$. If $d_{4}^{2} \equiv 2 \bmod 4$, then $n_{2}^{2} \equiv 0 \bmod 4$ and hence $4 \mid d_{4}^{2}$, a contradiction. Therefore, 
$4 \mid d_{4}^{2}$, and so $n_{2}$ must be odd. Now, we find

$$
4 \mid \frac{d_{4}^{2}}{d_{1}^{2} n_{2}^{2}}=\frac{1}{d_{1}^{2} n_{2}^{2}}+\frac{1}{n_{2}^{2}}+\frac{2}{d_{1}^{2}} .
$$

This forces $n_{2}^{2}=d_{1}^{2}=1$ and hence $d_{2}^{2}=d_{4}^{2}=4$ and $\operatorname{dim} \mathcal{C}=16$. Thus, $G=G(\mathcal{C})$ has order 4 and the homogeneous component $\mathcal{C}_{\psi}$ has $\operatorname{dim} \mathcal{C}_{\psi}=4$, for all $\psi \in \hat{G}$. In particular, $\left|\operatorname{Irr}\left(\mathcal{C}_{\psi}\right)\right|=1$ for any non-trivial character $\psi$ of $\hat{G}$. Therefore, $X_{4}$ must be fixed by $G$, contradicting $h \cdot X_{4}=X_{5}$.

Now, we have $\left(d_{1}, d_{3}, d_{4}^{2}\right) \equiv(0,0,1) \bmod 2$, we proceed to show that $\mathcal{C}$ is strictly weakly integral of $\operatorname{dim} \mathcal{C}=28$. Let $n_{i}=d_{i} / 2$ for $i=1,2,3$. Then $n_{i}^{2} \mid d_{4}^{2}$ and hence $n_{i}$ is odd for $i=1,2,3$. (5.1) becomes

$$
d_{4}^{2}=1+2 n_{1}^{2}+2 n_{2}^{2}+2 n_{3}^{2} .
$$

Now, this equation implies $d_{4}^{2} \equiv 7 \bmod 8$, since 1 is the only odd square modulo 8 . Therefore, $d_{4} \notin \mathbb{Z}$. Let $l=\operatorname{lcm}\left(n_{1}^{2}, n_{2}^{2}, n_{3}^{2}\right)$. Then $l$ is the square of an odd integer and hence $l \equiv 1 \bmod 8$. Since $n_{i}^{2} \mid d_{4}^{2}, m=\frac{d_{4}^{2}}{l} \equiv 7 \bmod 8$. Therefore,

$$
7 \leq m=\frac{d_{4}^{2}}{l}=\frac{1}{l}+2 \frac{n_{1}^{2}}{l}+2 \frac{n_{3}^{2}}{l}+2 \frac{n_{3}^{2}}{l} \leq 7 .
$$

This forces $n_{1}^{2}=n_{2}^{2}=n_{3}^{2}=l=1$. Hence $d_{1}=d_{2}=d_{3}=2, d_{4}^{2}=7$ and $\operatorname{dim} \mathcal{C}=28$.

From this we obtain:

Theorem 5.3. The only strictly weakly integral rank 7 categories are metaplectic categories.

Proof. Assume that $\mathcal{C}$ is strictly weakly integral of rank 7. By [19, Theorem 3.10] we have that 2||$G(\mathcal{C}) \mid$. Moreover, by Lemma 3.3, it follows that $\mid \overline{G(\mathcal{C}) \mid} \leq 5$. So there are two cases to consider: $G(\mathcal{C})=U(\mathcal{C}) \cong \mathbb{Z}_{2}$ or $|G(\mathcal{C})|=4$.

First suppose $\mathcal{D}:=\mathcal{C}_{p t}$ has rank 2. Clearly $\mathcal{D}$ is not modular, as 23 implies that $\mathcal{C}$ can have no modular subcategories. In particular $\mathcal{D}$ is premodular, and hence symmetric. If $\mathcal{D}$ is Tannakian, i.e. $\mathcal{D} \cong \operatorname{Rep}\left(\mathbb{Z}_{2}\right)$ then Prop. 5.2 implies that $\operatorname{dim} \mathcal{C}=28$ and we are done by Theorem 3.1. Otherwise $\mathcal{D} \cong \mathrm{sVec}$ and we have $\mathcal{C}_{a d}^{\prime}=\mathcal{D}$ hence $\mathcal{C}_{a d}$ is slightly degenerate $([16])$. In particular $\mathcal{C}_{a d}$ must have even rank by [16, Cor. 2.7]. It follow from [4] that $\mathcal{\mathcal { C }}_{a d}$ has dimension 10 , so $\operatorname{dim} \mathcal{C}=20$. This is impossible in rank 7.

Now if $|G(\mathcal{C})|=4$, consider the possible categories $\mathcal{C}_{a d}=\mathcal{C}_{e}$ corresponding to the universal grading. Clearly $\mathcal{C}_{a d}$ has rank at least 2 and at most 4 (by the pigeonhole principle) and even dimension. The classification of low-rank (integral) ribbon categories [4, 29,31] gives possible simple dimensions in $\mathcal{C}_{a d}$ as: $\{1,1\},\{1,1,2\},\{1,1,1,1\}$ and $\{1,1,2,2\}$, 
since the pointed subcateory of $\mathcal{C}_{a d}$ must have even dimension. If $\mathcal{C}_{a d}$ is pointed with rank 2 then one would have a rank 7 category of dimension 8 , which is absurd. In the second case the remaining three components must have dimension 6 , two of which contain one simple object of dimension $\sqrt{6}$. The other component must contain the remaining two invertible objects, which is impossible. If $\mathcal{C}_{a d}$ is pointed of rank 4 , the remaining three objects must lie in distinct components and each have dimension 2 . Such a category is not strictly weakly integral. In the last case we again have three remaining simple objects of equal dimension, since they reside in distinct components. But two of them are invertible, which is impossible.

It remains to consider integral modular categories of rank 7. For this we employ the methods developed on subsection 4.3 .

Lemma 5.4. Let $\mathcal{C}$ be a weakly integral modular category of rank $\leq 7$. If $p$ is an odd prime factor of $\mathrm{FSexp}(\mathcal{C})$ then $p \leq 7$ and $v_{p}(\operatorname{FSexp}(\mathcal{C}))=1$. If rank $\mathcal{C}=6$, $2<p \mid \operatorname{FSexp}(\mathcal{C})$ implies $p \leq 5$.

Proof. Suppose $\mathcal{C}$ is a weakly integral modular category of rank $\leq 7$ and $p$ is the largest odd prime factor of $\operatorname{FSexp}(\mathcal{C})$. Let $\sigma$ be a $p$-automorphism. Then, by Lemma 4.6 , $p \leq 13$. For any prime $p=5,7,11,13, v_{p}(\operatorname{FS} \exp (\mathcal{C})) \leq 1$ otherwise $\hat{\sigma}$ admits a support cycle of length $\left(p^{2}-p\right) / 2 \geq 10$ by Lemma 4.6 . This certainly won't happen in ranks $\leq 7$.

If $p=13$, then $\hat{\sigma}=(1,2,3,4,5,6)$ by Lemma 4.7 . Since the centralizer of $(1,2,3,4,5,6)$ in $S_{7}$ is $\langle(1,2,3,4,5,6)\rangle$, we get $\operatorname{Gal}(\mathcal{C})=\langle(1,2,3,4,5,6)\rangle$. However, this contradicts Theorem 4.8. Therefore, $p<13$.

If $p=11$, then $(1,2,3,4,5)$ is the unique $p$-support cycle of $\sigma$. Thus,

$$
\hat{\sigma}=(1,2,3,4,5) \text { or }(1,2,3,4,5)(0,6) .
$$

By Theorem 4.8, $\operatorname{Gal}(\mathcal{C})=\langle\hat{\sigma}\rangle$ implies $\operatorname{rank} \mathcal{C}=11$ or 9. Therefore, $\langle\hat{\sigma}\rangle \subsetneq \operatorname{Gal}(\mathcal{C})$ and $\operatorname{dim} \mathcal{C}=\frac{11 d_{1}^{4}}{4}$. Since the centralizer of $\langle\hat{\sigma}\rangle$ is $\langle(1,2,3,4,5),(0,6)\rangle, \operatorname{Gal}(\mathcal{C})=\langle(1,2,3,4,5),(0,6)\rangle$, and we have

$$
\frac{11 d_{1}^{4}}{4}=2+5 d_{1}^{2}
$$

However, the equation has no integral solution for $d_{1}^{2}$. Therefore, $p \leq 7$.

If rank $\mathcal{C}=6$ and $p=7$, then $\sigma$ has a unique support cycle $(1,2,3)$ and $\langle\hat{\sigma}\rangle \subsetneq \operatorname{Gal}(\mathcal{C})$ by Theorem 4.8 . Thus, $\operatorname{Gal}(\mathcal{C})=\langle(1,2,3),(0,5)\rangle$ or $\langle(1,2,3),(4,5)\rangle$. The second case 
implies $\mathcal{C}$ is integral and

$$
\frac{7 d_{1}^{4}}{4}=3 d_{1}^{2}+2 d_{4}^{2}+1
$$

by Theorem 4.8(i). The right hand side of this equation is an integer and we deduce that $2 \mid d_{1}$. Reduction modulo 2 now reveals the contradictory expression: $0 \equiv 1 \bmod 2$. Therefore, $\operatorname{Gal}(\mathcal{C})=\langle(1,2,3),(0,5)\rangle$ and

$$
\frac{7 d_{1}^{4}}{4}=3 d_{1}^{2}+d_{4}^{2}+2 .
$$

Next observe, that the Galois group moves 0 and hence $\mathcal{C}$ is strictly weakly integral. In this case, [19] implies that $\mathcal{C}$ is faithfully graded by an elementary abelian 2-group with the trivial component being given by the integral subcategory. So by dimension count we see that either $d_{1}$ or $d_{4}$ is and integer and the other dimension is not. Of course, if $d_{4} \notin \mathbb{Z}$, then it corresponds to a unique object of non-integral dimension and so Lemma 3.3 implies that $\mathcal{C}$ is an Ising modular category, an impossibility. Therefore, $d_{4} \in \mathbb{Z}$ and $d_{1} \notin \mathbb{Z}$. So $\frac{7 d_{1}^{4}}{8}=3 d_{1}^{2}$ but this implies $d_{1}^{2}=0$ or $\frac{24}{7}$, a contradiction.

Lemma 5.5. Suppose $\mathcal{C}$ is a weakly integral modular category of rank 7 such that $d_{1}=d_{2}=d_{3} \leq d_{4}=d_{5}=d_{6}$. Then, one of the following statements holds:

(i) $\left(d_{1}, d_{4}\right)=(1,1)$ and $\operatorname{dim} \mathcal{C}=7$.

(ii) $\left(d_{1}, d_{4}\right)=(1, \sqrt{2})$ and $\operatorname{dim} \mathcal{C}=10$.

(iii) $\left(d_{1}, d_{4}\right)=(1,2)$ and $\operatorname{dim} \mathcal{C}=16$.

Proof. The assumption implies the equality

$$
\operatorname{dim} \mathcal{C}=1+3 d_{1}^{2}+3 d_{4}^{2} .
$$

Thus $d_{1}^{2}, d_{4}^{2}$ are relatively prime and so $d_{1}^{2} d_{4}^{2} \mid \operatorname{dim} \mathcal{C}$. Then

$$
d_{1}^{2} \mid \frac{\operatorname{dim} \mathcal{C}}{d_{4}^{2}}=\frac{1}{d_{4}^{2}}+3 \frac{d_{1}^{2}}{d_{4}^{2}}+3 \in \mathbb{Z}
$$

implies $\frac{\operatorname{dim} \mathcal{C}}{d_{4}^{2}} \leq 7$. In particular $d_{1}^{2} \leq 7$ and $d_{4}^{2} \mid\left(1+3 d_{1}^{2}\right)$.

Now if $\mathcal{C}$ is strictly weakly integral, we must have $d_{1}=1$ since $|U(\mathcal{C})| \neq 1$. In this case $d_{4}^{2}$ divides 4 so that non-integrality implies $d_{4}=\sqrt{2}$.

Otherwise, $d_{1} \in \mathbb{Z}$ implies $d_{1}=1$ or $d_{1}=2$. If $d_{1}=1$ and $d_{4} \in \mathbb{Z}$ then $d_{4} \in\{1,2\}$ since $d_{4}^{2} \mid$ 4. If $d_{1}=2$, we have $d_{4}^{2} \mid 13$, which is impossible since $d_{4} \geq d_{1}$.

Thus the three possibilities are as in the statement. 
Remark 5.6. The proof of this lemma reveals that if $\mathcal{C}$ is a non-pointed weakly integral modular category and $d_{\max }^{2}=\max _{i} d_{i}^{2}$, then $\frac{\operatorname{dim} \mathcal{C}}{d_{\max }^{2}}$ is a positive integer strictly less than $\operatorname{rank} \mathcal{C}$.

Proposition 5.7. If $\mathcal{C}$ is a weakly integral modular category of rank 7 and $7 \mid \operatorname{FS} \exp (\mathcal{C})$, then $\mathcal{C}$ is either pointed or $\operatorname{dim} \mathcal{C}=28$.

Proof. Assume $\mathcal{C}$ is not pointed. We first show that $\operatorname{Gal}(\mathcal{C})$ does not contain any permutation of type $(1,3,3)$ or $(1,6)$ with 0 fixed. Assume the contrary. Then $d_{1}=$ $d_{2}=d_{3}, d_{4}=d_{5}=d_{6}$ and we have

$$
\operatorname{dim} \mathcal{C}=1+3 d_{1}^{2}+3 d_{4}^{2} .
$$

By Lemma 5.5, $\mathcal{C}$ must be pointed, a contradiction.

Let $p=7, \sigma$ the $p$-automorphism of $\mathcal{C}$ and $C_{1}$ a $p$-support cycle of $\sigma$. Then, in view of Lemma 4.7, $\operatorname{ord}\left(C_{1}\right)=3$, say $C_{1}=(1,2,3)$. Since all the 7 -support cycles must have length $\geq 3, C_{1}$ is the unique 7-support cycle of $\sigma$ and all other disjoint cycles are of length less than 3. It follows from Theorem 4.8 that $\operatorname{dim} \mathcal{C}=\frac{7 d_{1}^{2}}{4}$ and $2 \mid d_{1}^{2}$. Moreover,

$$
\hat{\sigma} \in\{(1,2,3),(1,2,3)(4,5),(0,6)(1,2,3)(4,5) \text { or }(0,6)(1,2,3)\}
$$

up to renumbering the non-zero labels.

If $\hat{\sigma}$ were one of the first three cases, then $\langle\hat{\sigma}\rangle \neq \operatorname{Gal}(\mathcal{C})$ otherwise it will contradicts Theorem 4.8. Since $\operatorname{Gal}(\mathcal{C})$ is an abelian subgroup of $S_{7}$ containing $\hat{\sigma}$, there exists an element of the form $C_{1}^{i}(4,5),(0,6) C_{1}^{i}(4,5)$ in $\operatorname{Gal}(\mathcal{C})$, for some $i=1,2$. In particular, we have the equation

$$
\frac{7 d_{1}^{4}}{4}=1+3 d_{1}^{2}+2 d_{4}^{2}+d_{6}^{2}
$$

with $2 \mid d_{1}^{2}$.

We claim that if the dimensions the simple objects of $\mathcal{C}$ satisfy (5.5), then $\mathcal{C}$ is a prime modular category of dimension 28. We first observe that the equation (5.5) modulo 2 implies that the parities of $\frac{d_{1}^{2}}{2}$ and $d_{6}^{2}$ are opposite. Since $d_{6}^{2} \mid \frac{7 d_{1}^{4}}{4}, d_{6}^{2}$ must be odd and $d_{1}^{2} / 2$ is even. Thus,

$$
28 n_{1}^{2}=1+12 n_{1}+2 d_{4}^{2}+d_{6}^{2},
$$

where $d_{1}^{2}=4 n_{1}$ for some positive integer $n_{1}$. If $d_{6}^{2} \equiv 3 \bmod 4$, then $d_{6} \notin \mathbb{Z}$ and so there exists $\tau \in \operatorname{Gal}(\mathcal{C})$ which admits a transposition of the form $(0, j)$, and in particular, $d_{j}=1$. Thus, $j$ can only be 4 or 5 but this does not balance the equation (5.6) modulo 4. Therefore, $d_{6}^{2} \equiv 1 \bmod 4$ and hence $d_{4}^{2}$ is odd. 
Since $d_{\max }^{2} \mid \operatorname{dim} \mathcal{C}=28 n_{1}^{2}$ and $\frac{\operatorname{dim} \mathcal{C}}{d_{\max }^{2}}<7, d_{\max }^{2} \neq d_{1}^{2}$ and so $d_{\max }^{2}=d_{4}^{2}$ or $d_{6}^{2}$. In particular, $d_{\max }^{2}$ is odd. Thus, $\frac{\operatorname{dim} \mathcal{C}}{d_{\max }^{2}}=4$ or $d_{\max }^{2}=7 n_{1}^{2} \equiv 3 \bmod 4$. Hence, $d_{\max }^{2}=d_{4}^{2}$ and (5.6) becomes

$$
14 n_{1}^{2}=1+12 n_{1}+d_{6}^{2} .
$$

In particular, $d_{6}^{2}$ and $n_{1}$ are relatively prime. Since $d_{6}^{2} \mid 28 n_{1}^{2}$ and $d_{6}^{2} \equiv 1 \bmod 4, d_{6}^{2}=1$ and so

$$
7 n_{1}^{2}-6 n_{1}-1=0 \text {. }
$$

This equation forces $n_{1}=1$ and hence $\left(d_{1}^{2}, d_{4}^{2}, d_{6}^{2}\right)=(4,7,1)$. Therefore, $\mathcal{C}$ is a prime modular category of dimension 28 , and this proves the claim.

As a consequence of the preceding claim, $\hat{\sigma}=(0,6)(1,2,3)$. To complete the proof, it suffices to show that $\langle\hat{\sigma}\rangle=\operatorname{Gal}(\mathcal{C})$. If not, then $\operatorname{Gal}(\mathcal{C})$ contains $(4,5)$ and so the dimensions of $\mathcal{C}$ satisfy (5.5) again. This implies $\mathcal{C}$ is a prime modular category of dimension 28 but then $\operatorname{Gal}(\mathcal{C})=\langle\hat{\sigma}\rangle$, which yields to a contradiction.

Theorem 5.8. If $\mathcal{C}$ is an integral modular category of rank 7 , then $\mathcal{C}$ is pointed.

Proof. Let $\mathcal{C}$ be an integral modular category of rank 7 , and assume it is not pointed. By Lemma 5.4, the prime factors of $\operatorname{FSexp}(\mathcal{C})$ can only be 2,3,5,7. By the Cauchy Theorem [5], the prime factors of $\operatorname{dim} \mathcal{C}$ can only be $2,3,5,7$. In view of Proposition 5.7, it suffices to prove that $7 \mid \operatorname{dim} \mathcal{C}$. Equivalently, it enough to show none of 2,3 or 5 is a prime factor of $\operatorname{dim} \mathcal{C}$. In view of Proposition 5.2, it is not possible that only two of the these primes are factors of $\operatorname{dim} \mathcal{C}$. It suffices to show that $\operatorname{dim} \mathcal{C}=2^{a} 3^{b} 5^{c}$ with $a b c \geq 1$ is not possible.

Suppose $\operatorname{dim} \mathcal{C}=2^{a} 3^{b} 5^{c}$ with $a b c \geq 1$. Let $\sigma$ be a 5 -automorphism of $\mathcal{C}$, and $C_{1}$ a 5 support cycle of $\sigma$. We first show that length of $C_{1}$ must be 2 . If not, then $\operatorname{ord}\left(C_{1}\right)=4$ and so that:

$$
\operatorname{dim} \mathcal{C}=1+4 d_{1}^{2}+d_{5}^{2}+d_{6}^{2}
$$

where we have ordered the simple objects so that $C_{1}=(1,2,3,4)$. This equation modulo 2 implies

$$
0 \equiv 1+d_{5}^{2}+d_{6}^{2} \quad \bmod 2 .
$$

Without loss of generality, we may assume $d_{5}$ is odd and $d_{6}$ is even. In particular, $4 \mid \operatorname{dim} \mathcal{C}$ as $d_{6}^{2} \mid \operatorname{dim} \mathcal{C}$. However, we then find $0 \equiv 2 \bmod 4$, a contradiction. Therefore, the dimensions of $\mathcal{C}$ do not satisfy $(5.7)$, and so $\operatorname{Gal}(\mathcal{C})$ does not contain any permutation which admits a disjoint cycle of length $\geq 4$. In particular, $\operatorname{ord}\left(C_{1}\right)<4$.

Now, we may assume $C_{1}=(1,2)$ and proceed to show that this is a unique 5-support cycle of $\sigma$. Then $\hat{\sigma}=(1,2)(3,4)$ or $\hat{\sigma}=(1,2)(3,4)(5,6)$. However, if $\operatorname{Gal}(\mathcal{C})$ contains 
any permutation of type $(1,2,2,2)$ with 0 fixed, the we have:

$$
\operatorname{dim} \mathcal{C}=1+2 d_{1}^{2}+2 d_{3}^{2}+2 d_{5}^{2} \equiv 1 \bmod 2 .
$$

This is not possible since such a category is pointed by [7]. Moreover, it implies that $\operatorname{Gal}(\mathcal{C})$ does not contain any permutation of type $(1,2,2,2)$ with 0 fixed. In particular, $\hat{\sigma}=(1,2)(3,4)$.

If $(3,4)$ is also a 5 -support cycle of $\hat{\sigma}$ then, by Lemma 4.9 , the dimensions $d_{i}$ of $\mathcal{C}$ satisfy (5.7) or

$$
1+2 d_{1}^{2}+2 d_{4}^{2}+d_{5}^{2}+d_{6}^{2}=\frac{1}{4}\left(d_{1}^{4}+d_{4}^{4}+\epsilon d_{1}^{2} d_{4}^{2}\right), \text { with } 2 \mid d_{1}, d_{2} \text { and } \epsilon=0,1,-1 .
$$

We have shown that the dimensions $d_{i}$ of $\mathcal{C}$ do not satisfy (5.7). By considering (5.9) modulo 2, we may assume that $d_{5}$ is odd and $d_{6}$ is even. Modulo 4, (5.9) then becomes

$$
0 \equiv 1+d_{5}^{2} \bmod 4
$$

but this is impossible as $d_{5}^{2} \equiv 1 \bmod 4$. Therefore, $(3,4)$ is not a 5 -support cycle if $\hat{\sigma}=(1,2)(3,4)$. In particular, $(1,2)$ is the unique 5 -support of $\sigma$. By Theorem 4.8, we find $\operatorname{dim} \mathcal{C}=\frac{5 d_{1}^{4}}{4}$ and $d_{1}=2 n_{1}$, for some positive integer $n_{1}$.

Suppose there exists a permutation $\tau \in \operatorname{Gal}(\mathcal{C})$ which admits a cycle of length $\geq 2$ and disjoint from $(1,2)$, say $(3,4, \ldots)$. Then the $d_{i}$ must satisfy:

$$
20 n_{1}^{4}=1+8 n_{1}^{2}+2 d_{4}^{2}+d_{5}^{2}+d_{6}^{2} .
$$

Then $d_{5}$ and $d_{6}$ must have opposite parities, otherwise the left hand side of $(5.10)$ would be congruent to 1 modulo 2 . We may assume $d_{5}$ is odd and $d_{6}=2 n_{6}$ for some positive integer $n_{6}$. Now, 5.10 modulo 4 yields

$$
0 \equiv 2+2 d_{4}^{2} \quad \bmod 4 .
$$

This forces $d_{4}$ to be odd, and we have

$$
\operatorname{dim} \mathcal{C} \equiv 4+4 n_{6}^{2} \quad \bmod 8 .
$$

Thus, $n_{6}$ must be odd, $8 \mid \operatorname{dim} \mathcal{C}$, and so $n_{1}$ is also even. Let $d_{1}=4 m_{1}$ for some positive integer $m_{1}$. Now, $\frac{\operatorname{dim} \mathcal{C}}{d_{j}^{2}}=\frac{2^{6} \cdot 5 m_{1}^{4}}{d_{j}^{2}}>7$ for $j=1, \ldots, 6$. But this contradicts that $\frac{\operatorname{dim} \mathcal{C}}{d_{\max }^{2}}<7$, see Remark 5.6 . Therefore, no permutation $\tau \in \operatorname{Gal}(\mathcal{C})$ admits a nontrivial cycle disjoint from $(1,2)$. Hence, $\operatorname{Gal}(\mathcal{C})=\langle\hat{\sigma}\rangle$ and $\hat{\sigma}=(1,2)$, which leads to a contradiction of Theorem 4.8 . 


\section{REFERENCES}

[1] B. Bakalov and A. Kirillov, Jr. Lectures on tensor categories and modular functors, volume 21 of University Lecture Series. American Mathematical Society, Providence, RI, 2001.

[2] M. Barkeshli, P. Bonderson, M. Cheng and Z. Wang. Symmetry, defects, and gauging of topological phases. Preprint arXiv:1410.4540 (2014).

[3] A. Bruguieres Catégories prémodulaires, modularisations et invariants des variétés de dimension 3 Math. Ann. 316 no. 2, 215-236 (2000).

[4] P. Bruillard. Rank 4 premodular categories, preprint arXiv:1204.4836 (2012).

[5] P. Bruillard, S.-H. Ng, E. Rowell, and Z. Wang. Rank-finiteness for modular categories to appear in J. Amer. Math. Soc. preprint arXiv:1310.7050 (2013).

[6] P. Bruillard, S.-H. Ng, E. Rowell, and Z. Wang. On classification of modular categories by rank, preprint arXiv:1507.05139 (2015).

[7] P. Bruillard and E. Rowell. Modular categories, integrality and Egyptian fractions. Proc. Amer. Math. Soc., 140 no. 4, 1141-1150 (2012).

[8] S.-X. Cui, C. Galindo, J. Plavnik and Z. Wang. On Gauging Symmetry of Modular Categories, preprint arXiv: 1510.03475

[9] S.-X. Cui and Z. Wang. Universal quantum computation with metaplectic Anyons, J. Math. Phys. 56, $032202(2015)$.

[10] A. Davydov, M. Müger, D. Nikshych, and V. Ostrik. The Witt group of non-degenerate braided fusion categories. J. Reine Angew. Math. 677, 135-177 (2013).

[11] Pierre Deligne, Catégories tannakiennes, The Grothendieck Festschrift, Vol. II. Progr. Math. 87, 111-195. Birkhäuser Boston (1990).

[12] C. Dong, X. Lin, and S.-H. Ng. Congruence property in conformal field theory, to appear in Algebra Number Theory, preprint arXiv:1201.6644 (2012).

[13] V. Drinfeld, S. Gelaki, D. Nikshych, and V. Ostrik. Group-theoretical properties of nilpotent modular categories, preprint arXiv:0704.0195 (2007).

[14] V. Drinfeld, S. Gelaki, D. Nikshych, and V. Ostrik. On braided fusion categories I. Selecta Math., 16(1):1-119 (2010).

[15] P. Etingof and S. Gelaki. Some properties of finite-dimensional semisimple Hopf algebras. Math. Res. Lett, 5:191-197 (1998).

[16] P. Etingof, D. Nikshych, and V. Ostrik. Weakly group-theoretical and solvable fusion categories. Adv. Math., 226(1):176-205 (2011).

[17] P. Etingof, D. Nikshych, and V. Ostrik. On fusion categories. Ann. of Math., 162(2):581-642 (2005).

[18] S. Gelaki, D. Naidu, and D. Nikshych. Centers of graded fusion categories. Algebra Number Theory, 3:959-990 (2009).

[19] S. Gelaki and D. Nikshych. Nilpotent fusion categories. Adv. Math., 217:1053-1071 (2008).

[20] C. Kassel. Quantum Groups, Graduate Texts in Mathematics 155, Springer-Verlag, New York, 1995.

[21] A. Kitaev. Anyons in an exactly solved model and beyond. Annals of Physics 321.1, 2-111 (2006).

[22] M. Müger. Galois theory for braided tensor categories and the modular closure. Adv. Math., 150(2):151-201 (2000).

[23] M. Müger. On the structure of modular categories. Proc. London Math. Soc., 87(2):291-308 (2003). 
[24] S. Natale. Faithful simple objects, orders and gradings of fusion categories. Algebr. Geom. Topol., 3(13):1489-1511 (2013).

[25] M.-B. Hastings, C. Nayak, and Z. Wang. On metaplectic modular categories and their applications. Comm. Math. Phys. 330, no. 1 45-68 (2014).

[26] D. Naidu and E. Rowell. A finiteness property for braided fusion categories. Algebr. Represent. Theory 15 no. 5, 837-855 (2011).

[27] S.-H. Ng and P. Schauenburg. Frobenius-Schur indicators and exponents of spherical categories. Adv. Math., 211(1):34-71 (2007).

[28] S.-H. Ng and P. Schauenburg. Congruence subgroups and generalized Frobenius-Schur indicators. Comm. Math. Phys. 300, no. 1, 1-46 (2010).

[29] V. Ostrik. Module categories, weak Hopf algebras and modular invariants. Transform. Groups, 8(2):177-206 (2003).

[30] V. Ostrik. Pre-modular categories of rank 3. Mosc. Math. J., 8(1):111-118 (2008).

[31] Viktor Ostrik. On fusion categories of rank 2. Math. Res. Lett., 10(2-3):177-183 (2003).

[32] D. Tambara and S. Yamagami, Tensor categories with fusion rules of self-duality for finite abelian groups, J. Algebra 209, no. 2, 692-707 (1998).

E-mail address: pjb2357@gmail.com

Pacific Northwest National Laboratory, 902 Battelle Boulevard, Richland, WA U.S.A

E-mail address: cn.galindo1116@uniandes.edu.co

Departamento de Matemáticas, Universidad de los Andes, Bogotá, Colombia.

E-mail address: rng@math.1su.edu

Department of Mathematics, Louisiana State University, Baton Rouge, LA U.S.A.

E-mail address: julia@math.tamu.edu

Department of Mathematics, Texas A\&M University, College Station, TX U.S.A.

E-mail address: rowell@math.tamu.edu

Department of Mathematics, Texas A\&M University, College Station, TX U.S.A.

E-mail address: zhenghwa@icrosoft.com

Microsoft Research Station Q and Department of Mathematics, University of CaliFornia, SANTA Barbara, CA U.S.A. 